\title{
The European Integrated Tokamak Modelling (ITM) effort: achievements and first physics results
}

\author{
G L Falchetto ${ }^{1}$, D Coster ${ }^{2}$, R Coelho ${ }^{3}$, B D Scott ${ }^{2}$, L Figini $^{4}$, D Kalupin ${ }^{5}$, E Nardon ${ }^{1}$, S Nowak ${ }^{4}$, \\ L L Alves $^{3}$, J F Artaud ${ }^{1}$, V Basiuk ${ }^{1}$, João P S Bizarro ${ }^{3}$, C Boulbe ${ }^{6}$, A Dinklage ${ }^{7}$, D Farina ${ }^{4}$, \\ B Faugeras $^{6}$, J Ferreira $^{3}$, A Figueiredo ${ }^{3}$, Ph Huynh $^{1}$, F Imbeaux $^{1}$, I Ivanova-Stanik ${ }^{8}$, T Jonsson $^{9}$, \\ H-J Klingshirn ${ }^{2}, \mathrm{C} \mathrm{Konz}{ }^{2}$, A Kus ${ }^{7}, \mathrm{~N}$ B Marushchenko ${ }^{7}$, G Pereverzevt,

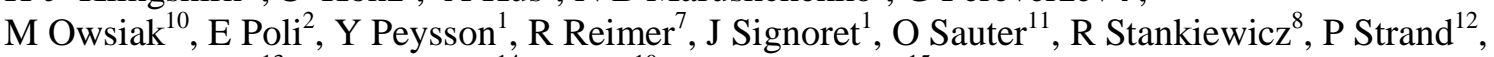 \\ I Voitsekhovitch $^{13}$, E Westerhof $^{14}$, T Zok $^{10}$, W Zwingmann ${ }^{15}$, ITM-TF contributors*, \\ the ASDEX Upgrade Team and JET-EFDA Contributors ${ }^{* *}$
}

1 CEA, IRFM, F-13108 Saint-Paul-lez-Durance, France.

2 Max-Planck-Institut für Plasmaphysik, EURATOM-IPP Association, Garching, Germany

3 Associação EURATOM/IST, Instituto de Plasmas e Fusão Nuclear, Instituto Superior Técnico, Universidade Técnica de Lisboa 1049-001 Lisboa, Portugal

4 Istituto di Fisica del Plasma CNR, Euratom-ENEA-CNR Association, 20125 Milano, Italy

5 EFDA-CSU Garching, Boltzmannstr. 2, D-85748, Garching, Germany

6 Univ. Nice Sophia Antipolis, Lab. JA Dieudonne, UMR 7351, F-06108 Nice 02, France

7 Max Planck Institut für Plasmaphysik, EURATOM Association, Greifswald, Germany

8 Institute of Plasma Physics and Laser Microfusion, EURATOM Association,00-908 Warsaw, Poland

9 Association EURATOM-VR, Fusion Plasma Physics, EES, KTH, SE-10037 Stockholm, Sweden

10 Poznan Supercomputing and Networking Center, IChB PAS, Noskowskiego 12/14, Poznan, Poland

11 Ecole Polytechnique Federale de Lausanne (EPFL), Centre de Recherches en Physique des Plasmas (CRPP), Association Euratom-Confederation Suisse, Lausanne, Switzerland

12 Department of Earth and Space Sciences, Chalmers University of Technology, Euratom-VR Association, SE-352 96 Göteborg, Sweden

13 EURATOM/CCFE Fusion Association, Culham Science Centre, Abingdon OX14 3DB UK

14 FOM Institute DIFFER, Association EURATOM-FOM, Nieuwegein, Netherlands

15 European Commission, Directorate-General for Research and Innovation, B-1049 Brussels, Belgium

e-mail of first author: gloria.falchetto@cea.fr

\begin{abstract}
A selection of achievements and first physics results are presented of the European Integrated Tokamak Modelling Task Force (EFDA ITM-TF) simulation framework, which aims to provide a standardized platform and an integrated modelling suite of validated numerical codes for the simulation and prediction of a complete plasma discharge of an arbitrary tokamak. The framework developed by the ITM-TF, based on a generic data structure including both simulated and experimental data, allows for the development of sophisticated integrated simulations (workflows) for physics application. The equilibrium reconstruction and linear MHD stability simulation chain was applied, in particular, to the analysis of the edge MHD stability of ASDEX Upgrade type-I ELMy Hmode discharges and ITER hybrid scenario, demonstrating the stabilizing effect of an increased Shafranov shift on edge modes. Interpretive simulations of a JET hybrid discharge were performed with two electromagnetic turbulence codes within ITM infrastructure showing the signature of trapped-electron assisted ITG turbulence. A successful benchmark among five EC beam/ray-tracing codes was performed in the ITM framework for an ITER inductive scenario for different launching conditions from the Equatorial and Upper Launcher, showing good agreement of the computed
\end{abstract}

\footnotetext{
* See the Appendix.

** See the Appendix of F. Romanelli et al., Proceedings of the 24th IAEA Fusion Energy Conference 2012, San Diego, US.
} 
absorbed power and driven current. Selected achievements and scientific workflow applications targeting key modelling topics and physics problems are also presented, showing the current status of the ITM-TF modelling suite. PACS 52.65

\section{Introduction}

The European Integrated Tokamak Modelling Task Force (ITM-TF) [1,2] aims at providing a standardized platform and an integrated modelling suite of validated numerical codes for the simulation and prediction of a complete plasma discharge in arbitrary tokamaks. In order to address such a challenge, the ITM-TF approach builds on a modelling infrastructure, focusing on the development of a data and communication ontology, i.e., standardizing the data exchange between different codes, through a generic data structure incorporating both simulated and experimental data. The elements of this data structure are identified as "Consistent Physical Objects", or CPO [3]. Physics modules of various complexities can be easily adapted to the data structure, which is code and language agnostic.

Thanks to the standardization of I/O through CPOs, physics modules can be seamlessly coupled into different integrated simulations (workflows), also, modules describing the same physics (eg equilibrium, transport modules, heating) can be easily interchanged within a same workflow, so to allow the physicist to choose and easily integrate the more appropriate model to tackle a specific physics problem. Moreover, in the ITM-TF framework all machine related data are extracted into standardized machine descriptions (MD) so that physics modules, like equilibrium reconstruction tools, also become independent of the specific tokamak experiment.

The ITM-TF uses the open-source Kepler ${ }^{\dagger}$ scientific workflow manager and orchestrator tool, which allows for a user-friendly graphical construction of the integrated simulation. Physics modules enter as actors of a Kepler workflow; all the data transfer among actors within a workflow occurs via CPOs. In Kepler, semantic types can be defined which allow to distinguish different CPOs and therefore verify whether a CPO output of an actor is correctly connected to the corresponding CPO input of the subsequent actor. Furthermore, the Kepler framework allows for interactive steering of simulations, through its capability to pause the simulation and alter some parameters; also, users can easily include actors for visualizing the present state of a simulation.

The ITM-TF uses the Kepler framework for simple run orchestration (workflows without convergence loops, named hereafter loosely coupled workflows) as well as more complicated workflows, involving mutual interactions among different codes (within loops, named hereafter tightly coupled workflows). It has to be noted, though, that the generic datastructure (CPOs) is totally independent of the used workflow orchestrator tool (Kepler or other), all the advantages of the generic datastructure remain if the physics modules are called in a classic Fortran workflow, with CPOs as arguments.

The framework developed by the ITM-TF has allowed for the development of sophisticated workflows for physics applications. Among those, the European Transport Simulator (ETS)[4] workflow, a leading ITM tool for both interpretive and predictive transport simulations and scenario modelling, incorporating a sophisticated module for synergy effects between heating schemes, several equilibrium modules, pellets, impurities, neutrals, sawteeth and neoclassical tearing modes (NTM) modules, as well as a variety of neoclassical and turbulence transport modules of different complexity. In this paper, selected achievements targeting key modelling topics and physics problems are outlined, showing the present status of the ITM-TF modelling suite. Besides, it is worth mentioning that the modules which can be coupled into ITM workflows can be either centrally distributed (residing on the common ITM Gateway cluster) or may be supplied by the user (in whichever programming language, including interpreted languages like Python and Matlab, provided they are CPO compliant independent modules).

First, we present applications of simple (loosely coupled) workflows. Physics results on the MHD equilibrium and linear stability of the plasma edge of ASDEX Upgrade and ITER hybrid scenario [5] as well as interpretive studies of a JET discharge using gyrofluid and gyrokinetic turbulence models

\footnotetext{
${ }^{\dagger} \mathrm{http} / / /$ kepler-project.org
} 
are reported in Section 2. We conclude Section 2.2, with an illustration of tightly coupled turbulencetransport workflows developed in the ITM framework.

The physics modules integrated into the different ITM workflows are being cross-verified within the ITM framework, as well as against existing integrated modelling codes to guarantee both their interchangeability and their validation. Results from a thorough benchmarking of electron cyclotron heating and current drive codes [6] on an ITER H-mode scenario for different launching conditions both from the Equatorial Launcher (EL) and Upper Launcher (UL) are shown in Section 3.1 Section 3.2 reports the ETS successful benchmarking against leading tokamak plasma core transport codes on a JET hybrid discharge [7]. In order to illustrate the flexibility and wide range of use cases for scientific workflows, Section 4 focuses on other relevant examples of tightly coupled workflows developed by the ITM-TF. Firstly, application of a direct coupling of the ETS core transport solver to a 2D edge transport code, demonstrated for the particular case of steady state and multiple impurities [8], is shown. The second example addresses the effect of NTMs on plasma transport and confinement, incorporated in ETS workflows via a dedicated NTM module that calculates the island frequency, width and associated reshaping in transport coefficients. Lastly, a successful proof-ofprinciple application of an ETS workflow including the coupling with a free-boundary equilibrium code, to the simulation of a VDE, is presented together with details on the coupling algorithm.

Finally, in Section 5 recent results are shown of the ongoing effort in ITM-TF to incorporate synthetic diagnostics [9] into the modelling framework (fusion products, 3D reflectometry, Motional Stark Effect, neutron and Neutral Particle Analyser), focusing on synthetic Motional Stark Effect spectra and comparison to the experimental data.

\section{Physics results}

A selection of some of the first physics results produced using the ITM-TF framework is presented in the following subsections.

\subsection{Equilibrium reconstruction and linear MHD stability}

The first demonstration of the use of ITM-TF integrated simulation workflows for physics studies on experimental data addressed equilibrium reconstruction, refinement and linear MHD stability calculations [5]. The corresponding Kepler workflow is illustrated in Figure 1, actors for freeboundary equilibrium reconstruction (e.g. EQUAL [10,11]), high resolution fixed-boundary GradShfranov solver (e.g. HELENA [12] or CHEASE [13]), and linear MHD stability (e.g. ILSA [14] or MARS-F [15]) are seamlessly integrated in the workflow environment. The machine independent equilibrium reconstruction code EQUAL developed within the ITM-TF has been extensively validated (at a first stage with magnetic data only) on JET discharges [16].

An analysis of the edge MHD stability of ASDEX Upgrade type-I ELMy H-mode discharges was carried out, using the stability chain coupling CLISTE, HELENA and ILSA [5]. CLISTE is a freeboundary equilibrium reconstruction code using input from poloidal field coil currents, magnetic and possibly kinetic plasma profile diagnostic measurements. The reconstructed coarse equilibrium is then passed to the high resolution reconstruction code HELENA and this refined equilibrium is used by the linear MHD stability code suite ILSA (in the particular case addressed here the ideal MISHKA code module of ILSA was used [13]).

Replacing the equilibrium actor with a JALPHA actor, which reads a previously calculated fixed boundary equilibrium from the database, modifies the pressure profile and/or the flux surface averaged current density and computes the new high resolution equilibrium, a j- $\alpha$ workflow is created. Stability diagrams can then be automatically computed using Kepler, by wrapping the linear $j-\alpha$ workflow in a double loop over the pressure and current scaling parameters. Computation times being substantial for such scans, the ITM-TF developed, in cooperation with the FP7 project EUFORIA [17], Kepler workflows for automatic job submission to Grid and Cloud infrastructures.

For pedestal height studies, the pressure and current density profiles in the edge can be scaled by a constant factor, while the core profiles are adapted to keep the plasma energy $\mathrm{W}_{\mathrm{MHD}}$ and the total plasma current Ip unchanged. For pedestal width studies, the widths of the pressure and current 
density pedestals can be scaled independently, again adjusting the core profiles such that $\mathrm{W}_{\mathrm{MHD}}$ and Ip remain the same. In this case, the pressure at the pedestal top and the amplitude of the bootstrap current remain constant, only the gradients change through variation of the width. Therefore, the total current flowing in the edge is smaller if the width is reduced.

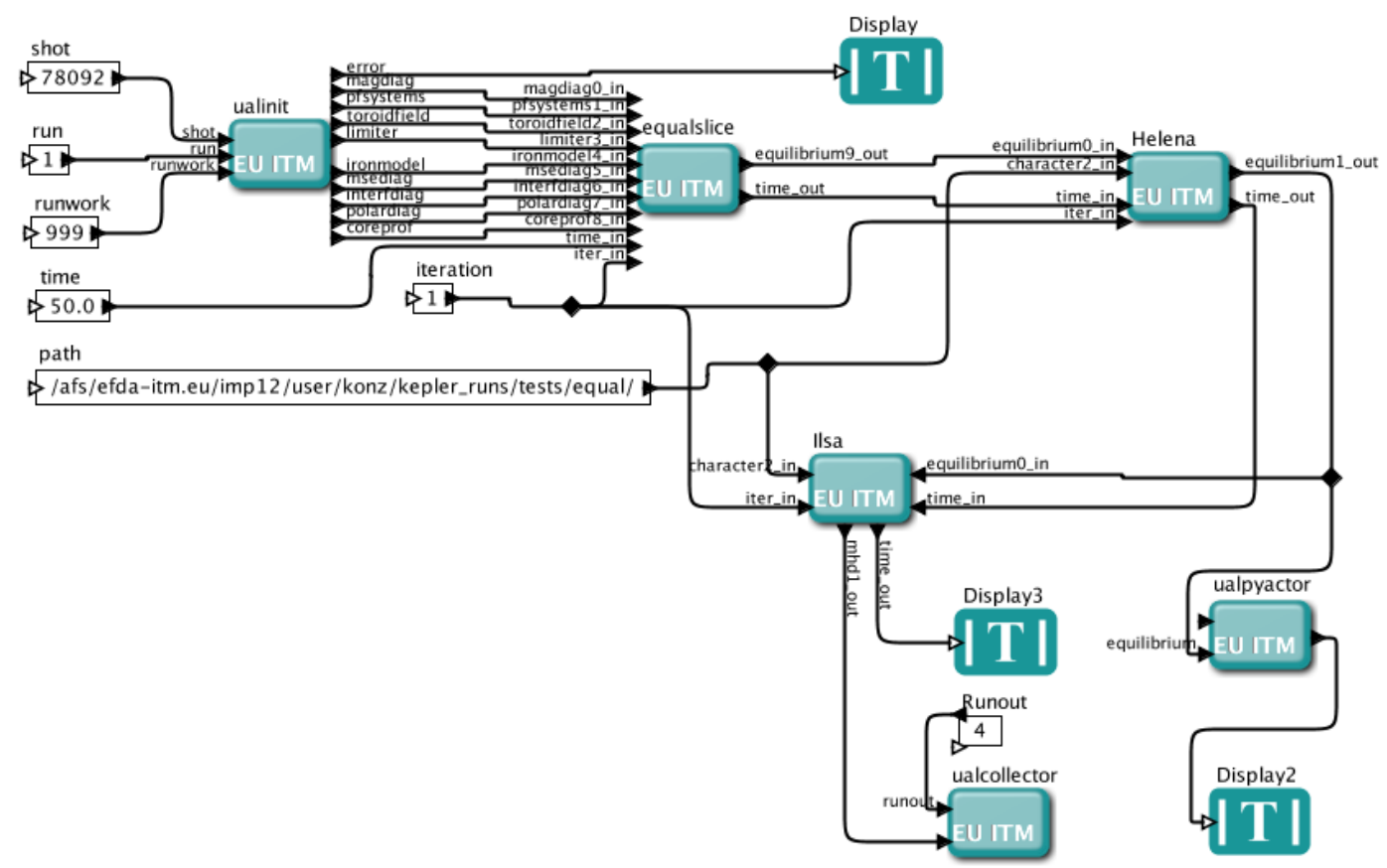

Figure 1 ITM-TF Kepler workflow for MHD linear stability coupling: an initialization module (ualinit) reading experimental data, EQUAL, HELENA and ILSA modules. A python script actor (ualpyactor) provides the visualization of the reconstructed equilibrium. Replacing equalslice with the j-alpha module allows to perform a parameter study by modifying pressure and plasma current.

Figure 2 shows the stability diagrams for the variation of the pedestal height and width for ASDEX Upgrade shot \#23223 at $\mathrm{t}=5.33 \mathrm{~s}$. The profiles were taken just before the crash of type-I ELMs. As expected, the experimental equilibrium is marginally unstable with a toroidal mode number $(\mathrm{n}=5)$ indicating a strong peeling component. Reducing the pedestal width, and thereby increasing the gradients, clearly drives the equilibrium unstable. It may also be noted (Figure 2 right) that the drive from the current density gradient (small bootstrap current width) dominates the drive from larger edge current (large bootstrap current width).
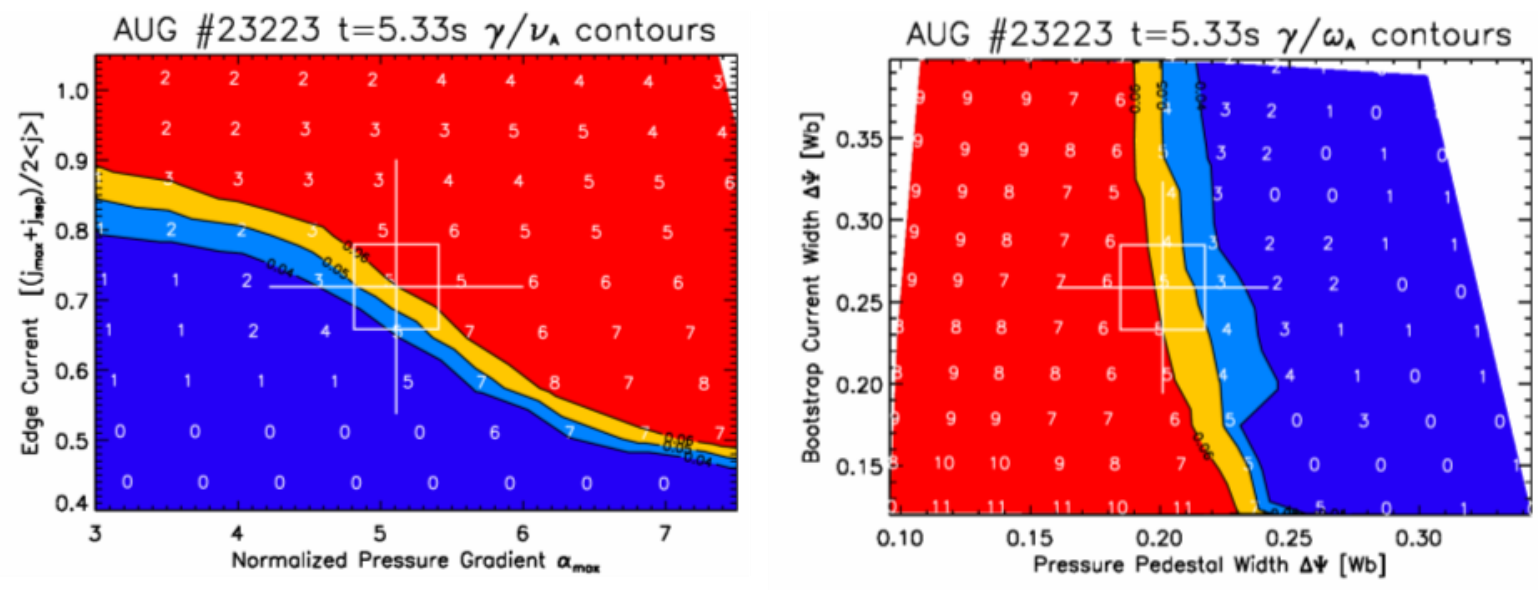

Figure 2 Pedestal height (left) and width (right) study for ASDEX Upgrade obtained with the J-alpha stability workflow [8]. The plot shows the contours of the linear ideal MHD growthrates $\gamma$ (normalized 
to the Alfvén frequency $v_{A}$ ) of the fastest growing edge modes (toroidal mode numbers are indicated by the white integers) in the plane defined by the maximum normalized edge pressure gradient $\alpha_{\max }$ and the normalized edge current density. Contours indicate the level of the diamagnetic drift frequency separating the stable (blue) from the unstable (red) region. The crosshair indicates the experimental equilibrium including error bars.

Core and pedestal scans of the normalized plasma beta $\beta_{\mathrm{N}}$ (applying, respectively, a scaling factor only on the core pressure profile or on the full profile) were also performed using the linear MHD stability chain for the ASDEX Upgrade type-I ELMy shot \#20116 at $\mathrm{t}=3.59 \mathrm{~s}$ as well as for an ITER hybrid "scenario 2" (kinetic profiles of the used ITER scenario 2, for the reference $\beta_{\mathrm{N}}=1.8$, are shown in Figure 3).
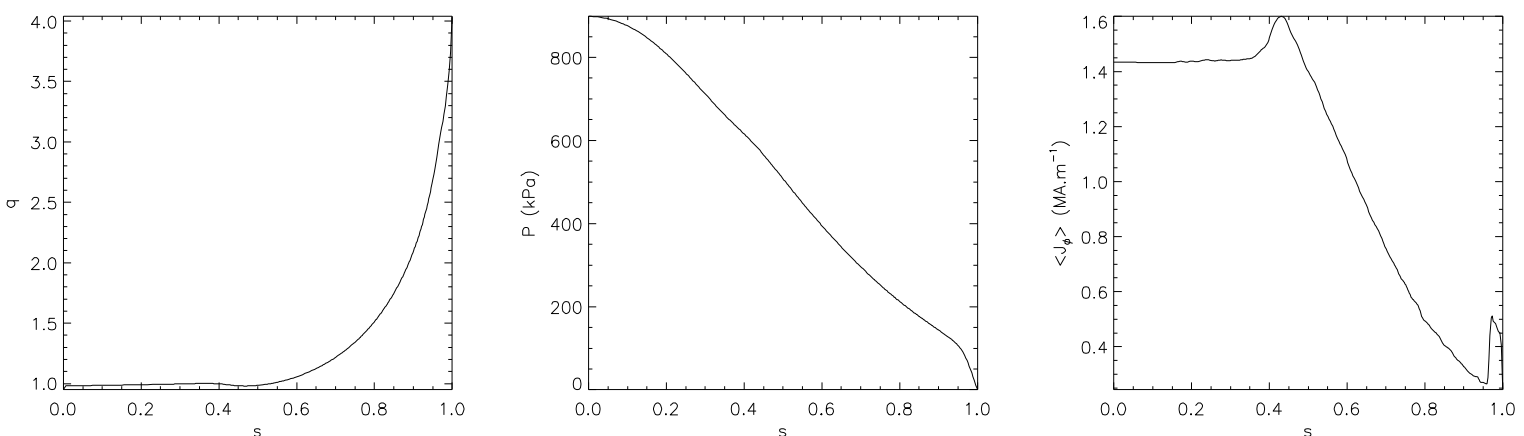

Figure 3 Safety factor, pressure and toroidal current profile of the used ITER scenario 2, for the reference $\beta_{N}=1.8$. The radial coordinate is $s=\operatorname{sqrt}\left(\psi / \psi_{\text {boundary }}\right)$.

The most unstable mode growth rates for the two scans are shown in Figure 4. It is evident from the computed growth rates in dashed lines that the increased Shafranov shift helps stabilizing edge modes (external kink modes of intermediate $n$ ). When scaling the entire pressure profile (solid lines), the destabilizing effect of the larger edge pressure gradient strongly dominates over the stabilizing effect by the Shafranov shift, inducing the destabilization of a (high $n$ ) ballooning mode. The ITER case shows a slight destabilization of a (low $n)$ pure peeling mode for large Shafranov shifts $\left(\beta_{N}>2.75\right)$.

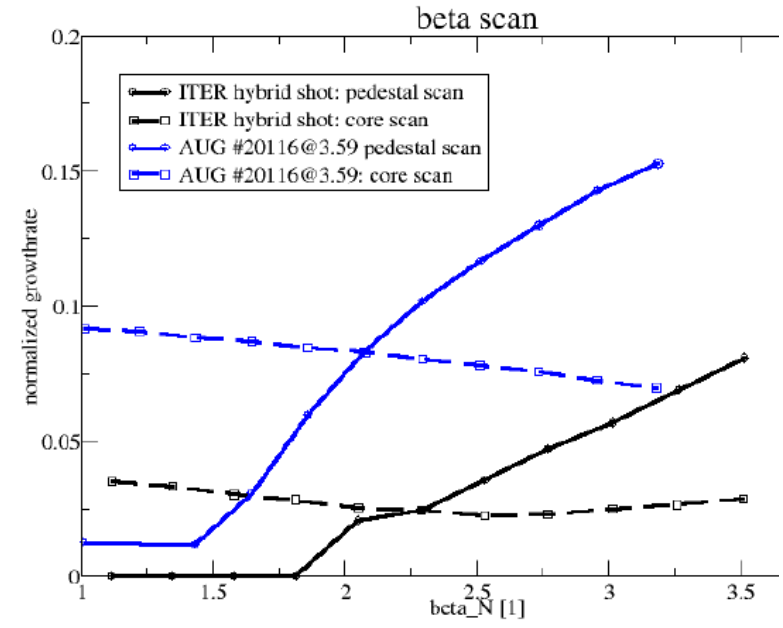

Figure 4 Core and pedestal scans of the normalized plasma beta for ASDEX Upgrade type-I ELMy shot \#20116 (blue) and an ITER hybrid scenario (black) [9]. The dashed lines show modification of the plasma $\beta_{N}$ via modification of the core pressure profile while keeping the pedestal pressure unchanged. The solid lines, on the other hand, show modification of the plasma $\beta_{N}$ via scaling of the entire pressure profile.

\subsection{Turbulence simulations}

A simple workflow allows conventional methods of comparing a turbulence code's transport results to experimental measurements and transport analysis. Run in a double-blind fashion, the result is almost always discrepant. Physical insight into the problem usually depends on diagnosing these discrepancies. A hybrid JET shot (\#77922) was used as a very interesting test-bed for radially local 
turbulence/transport computations, which happen to fail due to the set of parameters in the coreconfinement region (between 0.4 and 0.7 in normalized radius).

Discussions of the observed discrepancies among different turbulence/transport code simulations have highlighted several issues of provenance, namely what is used for the equilibrium flux surface structure, and what is used to define the dimensionless parameters of the runs (in this case, gradients). The profiles of the case under study turned out to be close enough to stability thresholds that small differences in magnetic shear or in the choice of radial coordinates (eg, $\rho_{\text {tor }}$ versus the midplane-cut minor radius) are enough to make the difference between stability and weak turbulence.

The prescribed case was profile data from JET shot 77922 at time $47.7 \mathrm{sec}$. The input data were provided by TRANSP [18] in interpretive mode from the actual experimental data which determine the profiles. Profiles of the electron density, electron and ion temperatures (ions hotter), and the toroidal current and pitch parameter $\mathrm{q}$ are shown in Figure 5.
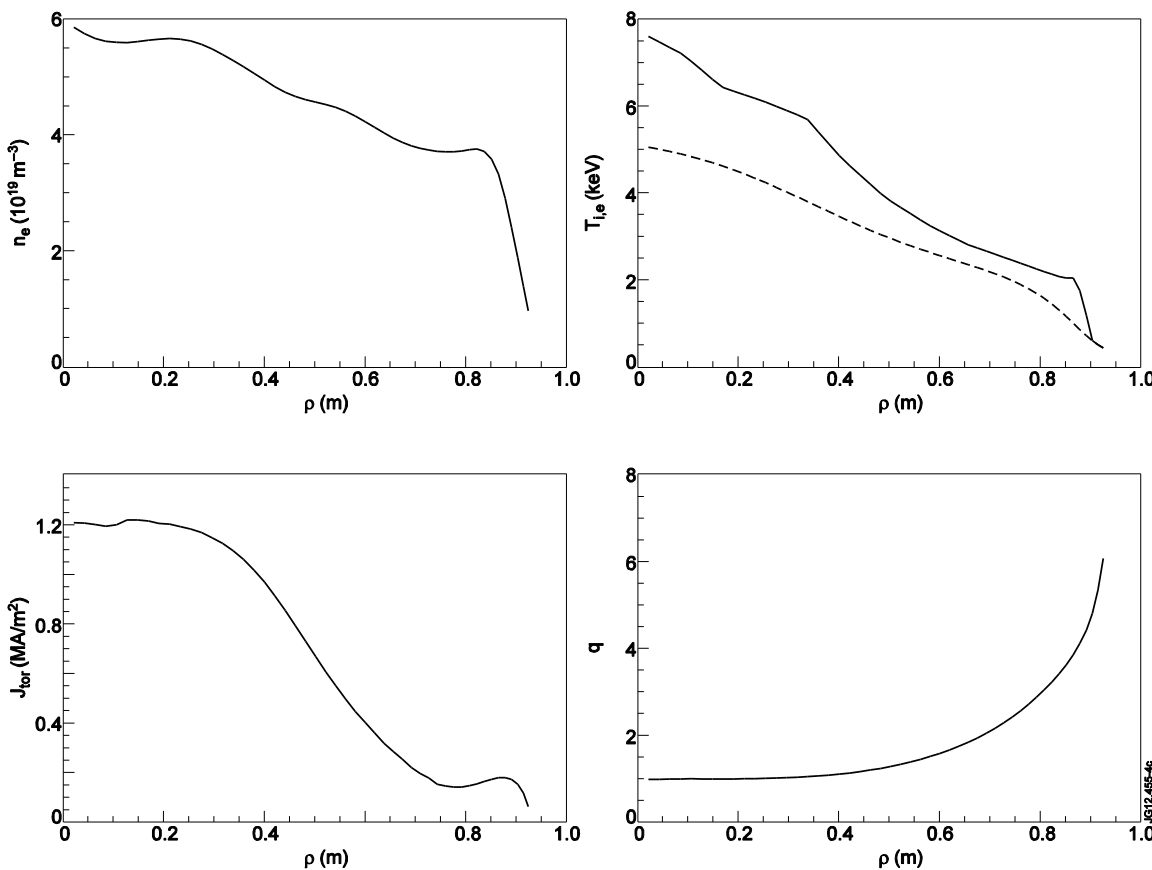

Figure 5 Profiles of the electron density, electron (dashed) and ion temperatures (full line), toroidal current and pitch parameter q, for the JET shot 77922 at 47.7 seconds given by the TRANSP interpretation through coreprof and equilibrium CPOs in the database case as discussed in the text.

The case is read from the database into coreprof and equilibrium CPOs, and then fed to the rest of the workflow, represented in Figure 6. Since the equilibrium_CPO did not contain sufficient information as needed by the successive flux-tube turbulence code modules (namely the pressure profile and the straight-field-line coordinate metric were missing), the workflow consisted of three actors: EQUPDATE which constructs equilibrium profile inputs for pressure and toroidal current from coreprof_CPO, and passes the equilibrium boundary surface, in this case the experimental separatrix, then the fixed-boundary Grad-Shafranov solver GKMHD which also fills the coord_sys element in the equilibrium_CPO, and then the turbulence code GEM, a fluxtube gyrofluid model [19]. GEM actor is executed in batch on HPC-FF, running in parallel one fluxtube at each of 0.4, 0.5, 0.6, and 0.7 normalised midplane-cut minor radius, it fills the coretransp_CPO and also provides the standard postprocess diagnostics for turbulence.

The same workflow was used replacing GEM with delta-FEFI, a delta-f gyrokinetic turbulence code (parent model to GEM otherwise similar in structure [20]), for direct comparisons between the two models.

The use of the GKMHD module was needed because a theoretical s- $\alpha$ model was found to be a very poor approximate to these experimental cases which are in the shaped geometry of a diverted tokamak. 
GKMHD sets up a regular triangular grid logically the same as placing flux surfaces onto nested hexagons. Each iteration consists of solving $-\Delta^{*} \psi=\mu_{0}\left\langle\mathrm{~J}_{\text {tor }} \mathrm{R}\right\rangle+\mu_{0}(\mathrm{dp} / \mathrm{d} \psi)\left(\mathrm{R}^{2}-\left\langle\mathrm{R}^{2}\right\rangle\right)$ where $\langle>$ denotes flux surface average, $p$ and $\left\langle\mathrm{J}_{\text {tor }} \mathrm{R}>/ \mathrm{R}_{0}\right.$ are the input profiles, and then moving the grid points towards or away from the axis such that the prescribed normalised $\psi$ of the surface agrees with the new values of $\psi(\mathrm{R}, \mathrm{Z})$. Otherwise it is a conventional Grad-Shafranov solver taking pressure and current on input. Afterwards, the resulting equilibrium_CPO is filled with coordinate metric information needed by fluxtube models. The midplane-cut minor radius is defined as $\left(\mathrm{r}_{\text {outboard }}-\mathrm{r}_{\text {inboard }}\right) / 2$ from the equilibrium_CPO; the normalised version is denoted as $r_{a}$ below.

Both GEM and delta-FEFI take the straight-field-line coordinate metric on input and construct a fieldaligned, shifted-metric coordinate system based on Hamada coordinates[21][22]. The fluctuations are initialised as a single Maxwellian density structure localised at nonlinear amplitude with Gaussian profiles to 10 ion sound gyroradii $\left(\rho_{\mathrm{s}}\right)$ in the drift plane and to $\mathrm{qR}_{0}$ along the field lines. The finite electron pressure launches shear-Alfven waves and then a drift wave field at nonlinear amplitude, and the system proceeds to fully developed turbulence unless it is nonlinearly stable [21][23].

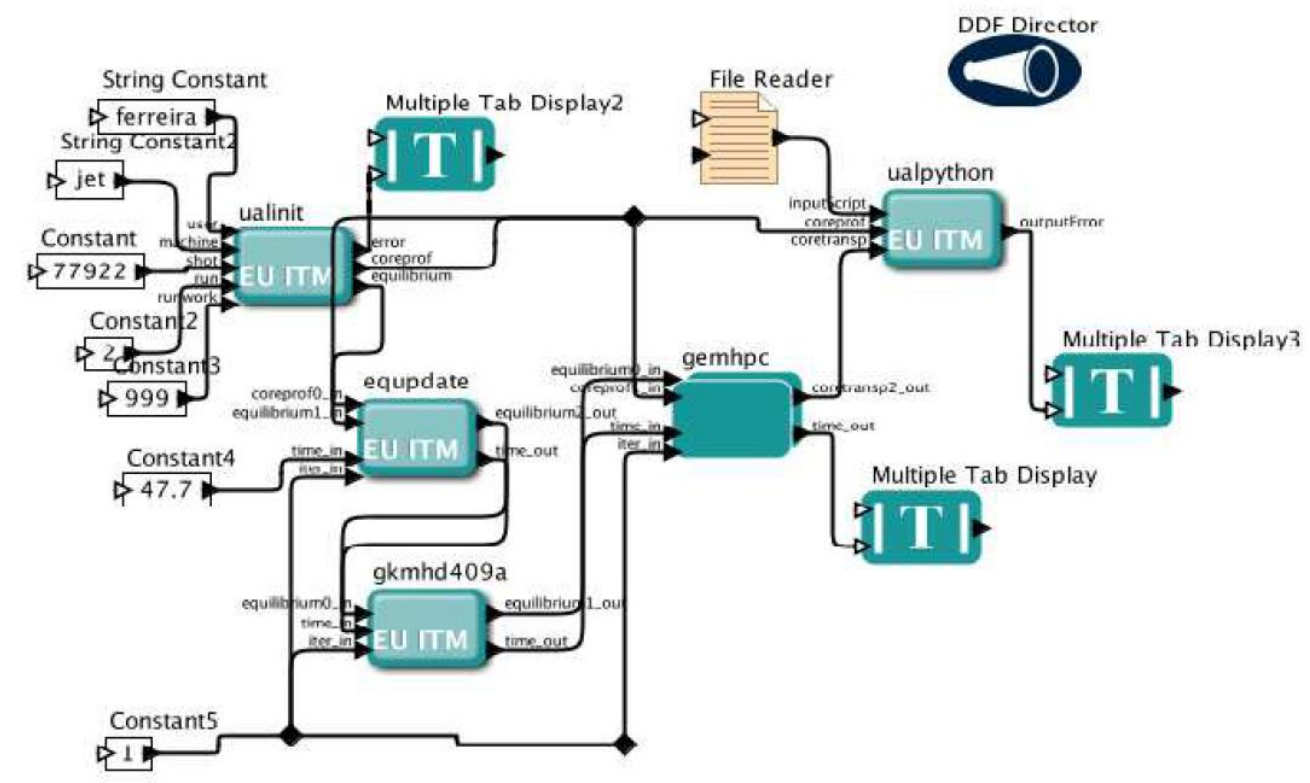

Figure 6 Turbulence workflow: JET shot data are read from the ITM database, the actor EQUPDATE provides equilibrium profile data and the equilibrium boundary, i.e. the separatrix, to actor GKMHD which adds the metrics information; both equilibrium_CPO and coreprof_CPO are input to the turbulence actor GEMHPC which runs in batch GEM gyrofluid flux-tube code in parallel on the HPC-FF.

Gyrofluid runs are held in saturation or decay for 4000 gyro-Bohm times $\left(\tau_{\mathrm{GB}}=\mathrm{L}_{\perp} / \mathrm{c}_{\mathrm{s}}\right.$, where $\mathrm{L}_{\perp}$ is the steepest gradient scale length and $c_{s}$ the ion sound speed). Gyrokinetic runs only went to $1000 \mathrm{~L}_{\perp} / \mathrm{c}_{\mathrm{s}}$ due to the far greater computational expense. Each fluxtube is an independent run, with its own normalised units including normalised time, $\tau_{\mathrm{GB}}$ being different for each case. The timestep is $0.002 \tau_{\mathrm{GB}}$, allowing for extreme transients which are found in the early stages of some core-parameter cases. The domain size is $20 \pi \rho_{\mathrm{s}}$ in the radial direction, $80 \pi \rho_{\mathrm{s}}$ in the drift-angle direction, and one connection length $2 \pi \mathrm{qR}$ in the parallel direction. The grid is $128 \times 128 \times 32$ in these directions, respectively. The numerical scheme is given in [19], mostly following [23]. Delta-FEFI uses the same scheme as GEM with the additional ingredient being the phase-space parallel bracket [20].

We concentrate on the case $r_{a}=0.6$ since both codes found stability or on-threshold behaviour at 0.7 . The normalised parameters (defined as in [24]) at $\mathrm{r}_{\mathrm{a}}=0.6$ are beta-hat $=0.38$, mu-hat $=0.022, \mathrm{C}=$ $3 \times 10^{-4}, \mathrm{~T}_{\mathrm{i}} / \mathrm{T}_{\mathrm{e}}=1.25, \mathrm{R} / \mathrm{L}_{\mathrm{Ti}}=6.30, \mathrm{~L}_{\mathrm{Ti}} / \mathrm{L}_{\mathrm{Te}}=0.68, \mathrm{~L}_{\mathrm{Ti}} / \mathrm{L}_{\mathrm{n}}=0.38$, and $\mathrm{qR}_{0} / \mathrm{L}_{\mathrm{Ti}}=9.0$.

GEM found a weak-to-stable ITG case at $r_{a}=0.6$ (dominant ExB/ion-gradient energetics), with ion heat flux $Q_{i}<0.1$ in gyro-Bohm units of $p_{e} c_{s}\left(\rho_{s} / L_{\perp}\right)^{2}$, whereas it showed stability at 0.4 , a very weak ITG 
case at 0.5 and approximately null growth at 0.7 . The delta-FEFI results are quite different: at $r_{a}=0.4$ and 0.5 , the code crashed apparently due to difficulty with the kinetic ballooning mode, KBM (deltaFEFI has never managed a saturated nonlinear-KBM case) whereas, interestingly, GEM had not found it; the $r_{a}=0.7$ case was definitively stable and the $r_{a}=0.6$ case produced what can be identified as a strongly trapped-electron enhanced ITG turbulence case, the evidence of which is worth showing.

Figure 7 shows the velocity space distribution of the contributions of delta-f to the turbulent ExB fluxes: all of the activity in the electrons and almost all in the ions is in the trapped domain (smaller $v_{/}$ for finite $\mu \mathrm{B}$ ). This is the clearest-possible identifier for a role of trapped electrons despite the iondominant energetics and is the basis for the named trapped-electron assisted ITG turbulence. It has to be mentioned that this result differs from that provided by GEM, as its gyrofluid model does not include trapped electrons [24].
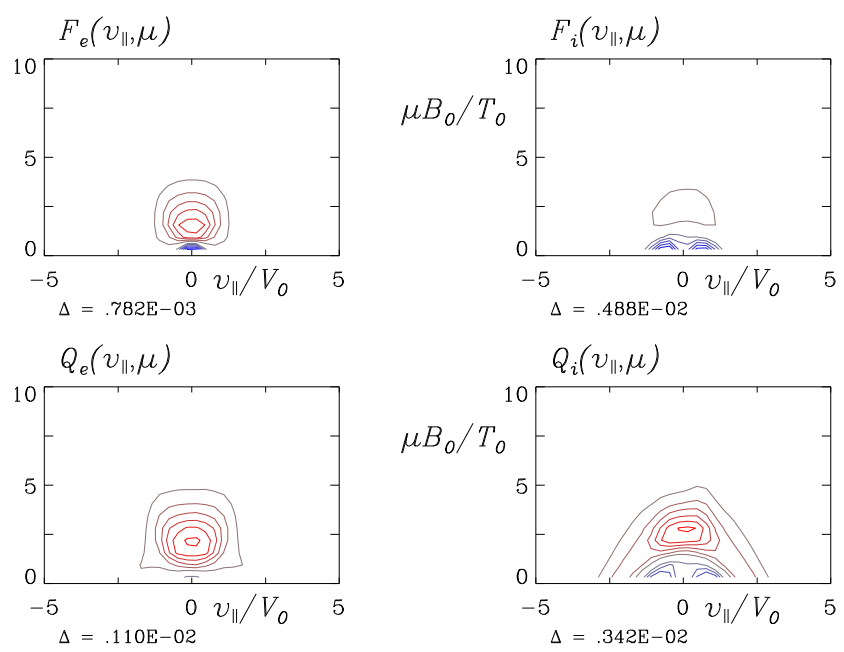

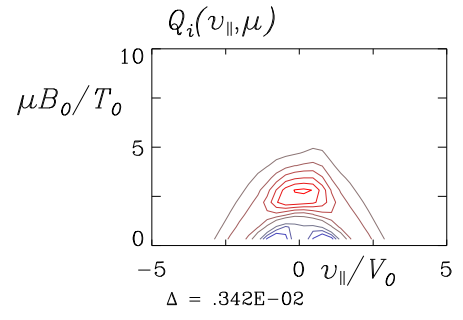

Figure 7 Velocity-space distribution of the ExB particle $(F)$ and heat fluxes $(Q)$ in the electrons and ions, in a snapshot at the end of the delta-FEFI run.

The trapped zone is roughly the 60-degree cone centred upon the vertical axis where $v_{/ /}=0$.

Almost all of the activity in the ions, and essentially all of it in the electrons, is in the trapped zone. These trapped-electron features together with the dominant ITG energetics (not shown) yield the description "trappedelectron enhanced ITG turbulence".

Besides the above simple workflow used for a transparent cross-benchmarking of turbulence modules producing the above physics results, tightly-coupled transport workflows were developed, wherein the turbulence module provides heat and particle diffusion coefficients to a transport solver, similar to the strategy used by other models ([25][26][27]). A demonstration of ITM progress towards turbulencetransport workflows with the ETS core transport solver is given in [28]. A parallel effort within a different scientific workflow framework is presented in [29].

Herein, we describe our progress in the coupling with an equilibration model which solves a statistical steady-state equilibrium rather than a time-dependent transport problem. This essentially replaces the time step loop with a convergence loop for the time-independent problem. Nevertheless, the term "time step" is convenient to the generic transport workflow structure (Figure 8).

The main Kepler workflow including the time loop is shown in Figure 8, in which the "workflow core" is a composite actor representing one time/convergence step of the transport workflow.

The sequence of operations of the workflow core, shown in Figure 9, is detailed in the following. Here, BPROFS is the transport equilibration model, used in place of the ETS module implemented in [28],[29]. 


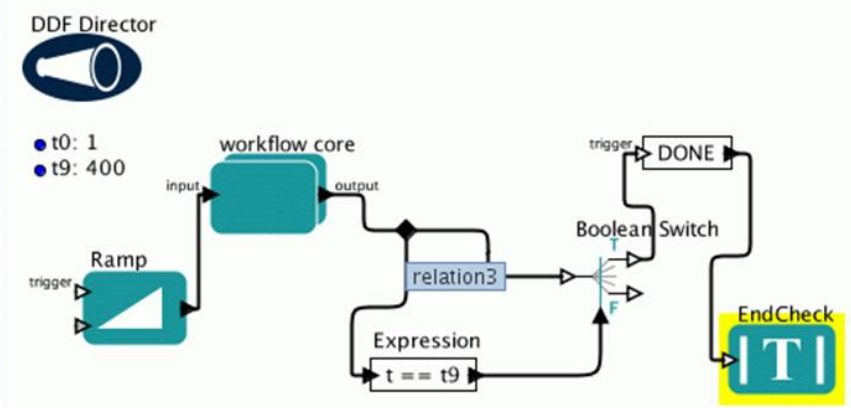

Figure 8 Topmost level Kepler workflow. The actor "ramp" corresponds to the control of the time loop (it generates integers from 1 to the maximum value of the time step). "workflow core" is a composite actor representing one time step of the transport workflow.

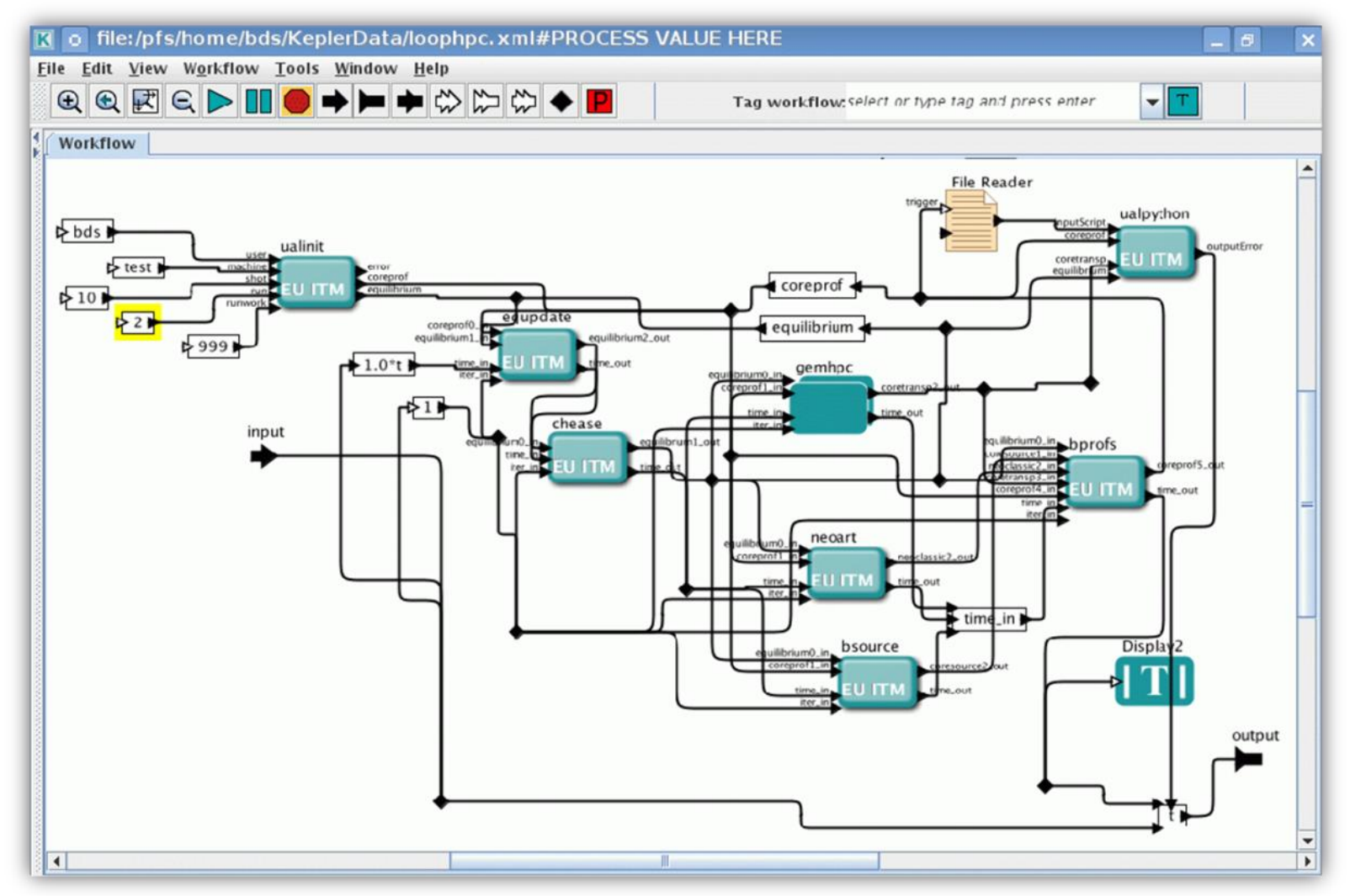

Figure 9 Workflow core, representing the sequence of operations performed during one time step of the transport workflow of Figure 8. Detailed description is given in the text.

The UALINIT actor is executed only once, at the beginning, to read the input data from the database; it provides the initial coreprof_CPO and equilibrium_CPO at the initial time (for time steps after the first one, the "coreprof" and "equilibrium" boxes shown at the top replace the UALINIT actor, representing the previous step's output ). At each time step the EQUPDATE actor sets up a new equilibrium_CPO using the pressure and current from the coreprof_CPO and the LCFS boundary from the equilibrium_CPO. This is then fed into the CHEASE actor to calculate a new, updated equilibrium_CPO. The coreprof_CPO and equilibrium_CPO are then used as inputs for the remaining actors: GEM, which provides a coretransp_CPO, NEOART, a neoclassical transport module providing a neoclassic_CPO, and BSOURCE, a simple analytical source model which provides a coresource_CPO. All of these are fed into the BPROFS actor, which updates the coreprof_CPO according to a simplified profile-equilibration model using running exponential averages [30] of the transport to relax the profiles into a state of transport equilibrium (the aim is not a transport simulation 
but a procedure to find a steady state). Review information and detailed comparison of relaxation methods can be found in [31].

The only parallel actor is GEMHPC, the first call to which launches a batch job on the HPC-FF, consisting of 8 fluxtubes on the profile, arranged as in the single-run case described above, on 1024 cores. The HPC-FF job runs one segment of $10 \tau_{\mathrm{GB}}$, returns a coretransp_CPO, and waits. Subsequent loop steps "fire" the actor again, and it sends a message to the job containing the coreprof_CPO and equilibrium_CPO and instructions to evolve GEM's state for another $10 \tau_{\mathrm{GB}}$ and return running exponential averages of the turbulent flux profiles to the coretransp_CPO, after which the job waits again and the GEMHPC actor sends the coretransp_CPO on to the BPROFS actor.

The batch job is kept running until it either crashes or accepts a stop signal from the workflow indicating completion - that is, the batch job needs only be submitted and wait in the queue once.

This workflow was applied to a JET-sized case with a model LCFS boundary for ITER (i.e., the R and $\mathrm{Z}$ are halved). A power source of $10 \mathrm{MW}$ for each species was specified by the BSOURCE actor. The loop ran for 120 time steps (close to a relaxation time for many cases). The main transport workflow ran on the ITM Gateway cluster, while GEM's batch job ran on the HPC-FF on 1024 cores.

Temperature profile modifications induced by the turbulence coefficients occurred only in the edge, producing a fast profile steepening (the core being marginally stable and turbulence delayed), that eventually crashed the equilibrium reconstruction. Figure 10 shows the outcome of the python visualization actor (see Figure 9 top-right), which allows the monitoring of the simulation at each transport time step during the workflow.

This is work in progress, as the workflow scheme is only mature and robust for $s$ - $\alpha$ model cases actually running only GEM and BPROFS by themselves.

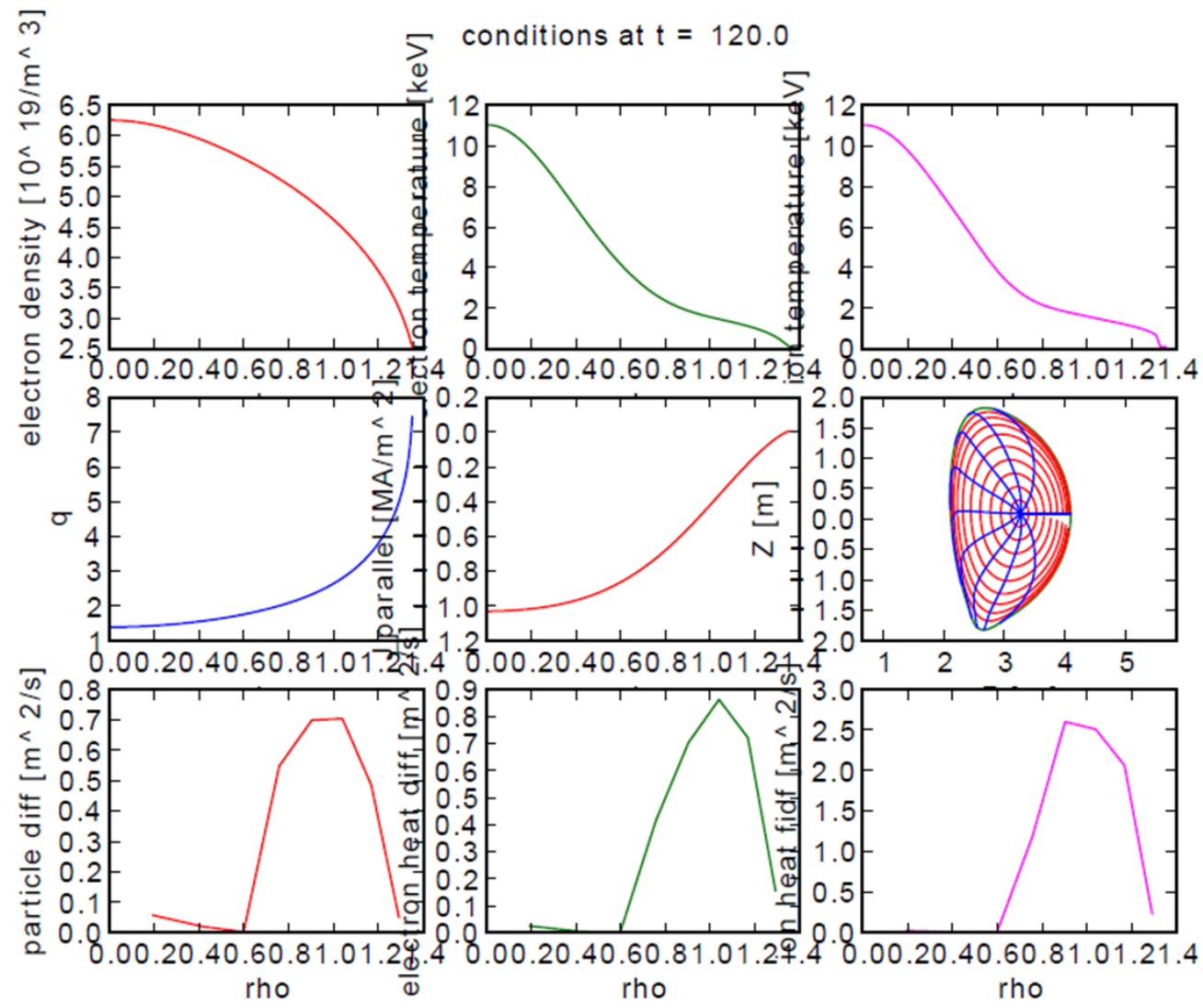

Figure 10 Instantaneous profiles and magnetic equilibrium after 120 time steps of the coupled turbulencetransport workflow (outcome of the ualpython visualization actor, see Figure 9). Top and middle row, from left to right: electron density (not evolved), ion and electron temperature, safety factor profile, parallel current (coreprof_CPO); magnetic equilibrium (equilibrium $C P O$ ). Bottom row: particle, electron heat and ion heat diffusivities (coretransp_CPO). 


\section{Verification and validation}

The ITM-TF framework is a valuable environment for a rigorous cross-verification of codes describing the same physics processes with different models, since by interchanging those as modules within the same workflow the possible external sources of discrepancy are minimized. Considering the fundamentals of an integrated transport simulator, it is essential to address the benchmarking of the equilibrium and core profile evolution solvers as well as the transport and turbulence or heating and current drive modules. Equilibrium codes went through benchmarking both within the ETS workflow [25] and independently, whereas turbulence and MHD codes cross-verification is ongoing on specified test cases within dedicated workflows. In this section the benchmarking of standalone electron cyclotron heating and current drive codes on an ITER scenario as well as. the ETS validation against existing integrated modelling transport codes on a JET hybrid discharge are presented. It has to be mentioned that the ETS was previously extensively verified [32][6]. The very good agreement achieved for the simulated quantities and applied modules lays the foundations for the use of ETS for both predictive and interpretative runs on present devices and ITER, in a variety of scenarios.

\subsection{Benchmarking of electron cyclotron heating and current drive codes on an ITER scenario}

A benchmark among five European EC beam/ray-tracing codes (C3PO [33], GRAY [34], TORAYFOM [35], TORBEAM [36], TRAVIS[37]) has been successfully performed [6] withhin the ITM framework for a standard inductive H-mode ITER scenario ("Scenario 2") at the end of burn phase, for three different launching conditions both from the Equatorial Launcher (EL) and Upper Launcher (UL), see Table I.

\begin{tabular}{lcccccc}
\hline Case & $R_{m}(\mathrm{~m})$ & $z_{m}(\mathrm{~m})$ & $\alpha\left(^{\circ}\right)$ & $\beta\left(^{\circ}\right)$ & $w_{0}(\mathrm{~m})$ & $d(\mathrm{~m})$ \\
\hline EL25 & 9.27 & 0.62 & 0 & 25 & 0.030 & 0.00 \\
EL40 & 9.27 & 0.62 & 0 & 40 & 0.030 & 0.00 \\
UL & 6.90 & 4.18 & 48 & 18 & 0.021 & 1.62 \\
\hline
\end{tabular}

Table I Launching conditions used in the benchmark. The poloidal and toroidal launching angles are defined as $\alpha=\tan ^{-1}\left(k_{0, z} k_{0, R}\right)$ and $\beta=\sin ^{-1}\left(k_{0, \phi} / k_{0}\right)$, where $\left(k_{0, R}, k_{0, \phi}, k_{0, z}\right)$ are the cylindrical wave vector components of the launched wave. The beam has a Gaussian profile, with waist $w_{0}$ at a distance $d$ from the launching point. The model here considered corresponds to simple astigmatism, when the spot ellipse and the curvature ellipse are rotated by the same angle.

The three cases have been selected to cover different geometries and physics of interest in ITER: injection of divergent beams from the EL, either absorbed in the core at quite large $T_{e}$ (EL25), or characterized by quite long trajectories and large $N_{/ /}$(EL40), and of a highly focused beam that drives the EC current in a narrow channel (UL). The frequency of the launched beam is $170 \mathrm{GHz}$ and the input power is $1 \mathrm{MW}$. Figure 11 represents the used plasma equilibrium and beam trajectories.

Among these codes, GRAY, TORBEAM and TORAY-FOM had participated also in the benchmark exercise in [38] that was run on the same ITER Scenario using only a divergent Gaussian beam launched from the UL. Since then, the codes have been modified and updated to include different physics modules as, e.g., the current drive model.

The fact that the codes run in the same ITM workflow simplifies the verification and, at the same time, guarantees a more detailed check of the various steps of the benchmark. Note that any module can be switched into an ETS time-dependent simulation since they share the same interface through CPOs.

The steps taken in the benchmarking study consisted in: (i) an extensive check of matching between ITM's and all codes' coordinate and sign conventions as well as physical quantities definitions, to ensure that the input and output data were correctly interpreted and written by the codes; (ii) a comparison among the computed wave trajectories, with particular consideration of the vacuumplasma transition; (iii) a comparison of the power absorption and current drive results. 


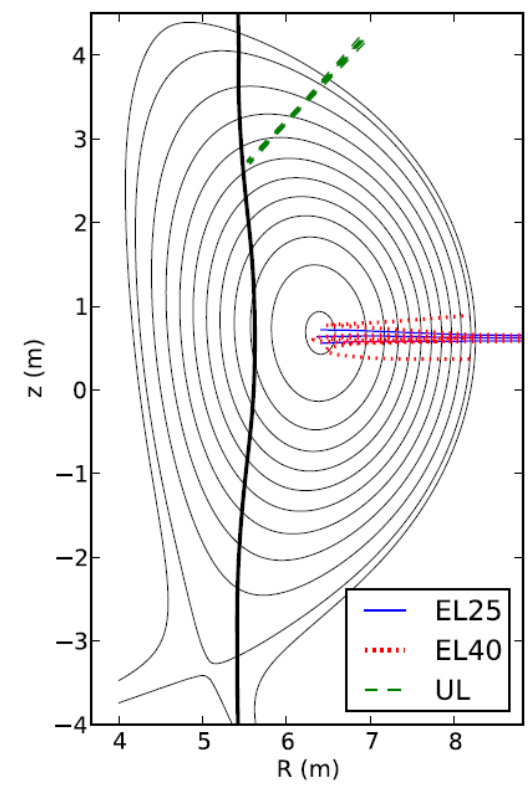

Figure 11 Magnetic equilibrium of the used ITER scenario 2. The cold resonance at $170 \mathrm{GHz}$ is shown (thick vertical line), with the beam-tracing computed by the GRAY code for the three considered launching (from [6] with kind permission of the European Physical Journal, EPJ).

Good agreement was found, with differences in total current $\left|\delta \mathrm{I}_{\mathrm{CD}} / \mathrm{I}_{\mathrm{CD}}\right|<15 \%$, and with peak values of power density $d P / d V$ and driven current density typically matching within $10 \%$, and the position of the profiles match within $\delta \rho \sim 0.02$ in normalized radius units (Figure 12, partially taken from [6]).

Small discrepancies can be ascribed to the different models used for wave propagation and absorption and current drive. The EL25 case considers a beam trajectory passing very close to the magnetic axis, where small differences in the interpolation of the equilibrium data or in the beam trajectory may result in relevant changes in the flux averaged power density $\mathrm{dP} / \mathrm{dV}$ value, as can be seen in the TORAY-FOM result at rho $<0.15$. In the EL40 case, Doppler broadening dominates the effect of finite beam size in the determination of the profiles width, and all the codes here agree very well. Some differences can also be appreciated among the results for the UL case, mainly because this case is more demanding in terms of spatial resolution required to reconstruct the actual shape of the absorption profile, since the focused beam considered here produces a much narrower (full width at 1/e $\Delta \rho \sim 0.015$ ) absorption profile than those obtained with the Equatorial Launcher. In this respect, the difference of C3PO/LUKE here results from a coarser grid considered in the calculation.

In the UL case, despite the focused beam, the profiles are reasonably well reconstructed also by raytracing codes, giving results comparable to those obtained by the codes which account for diffraction effects. The large edge density gradient, and long path from boundary to absorption region, amplifies the impact of edge refraction on beam propagation. Nonetheless, the influence of the observed discrepancies on computed power and current density profiles is still moderate. Only in case of strongly focused beam, like in the UL case, the uncertainty may approach the profiles width. Deeper analysis of the discrepancies among the different codes and underlying models used for wave propagation, absorption and current drive is ongoing and will be presented in a following publication.
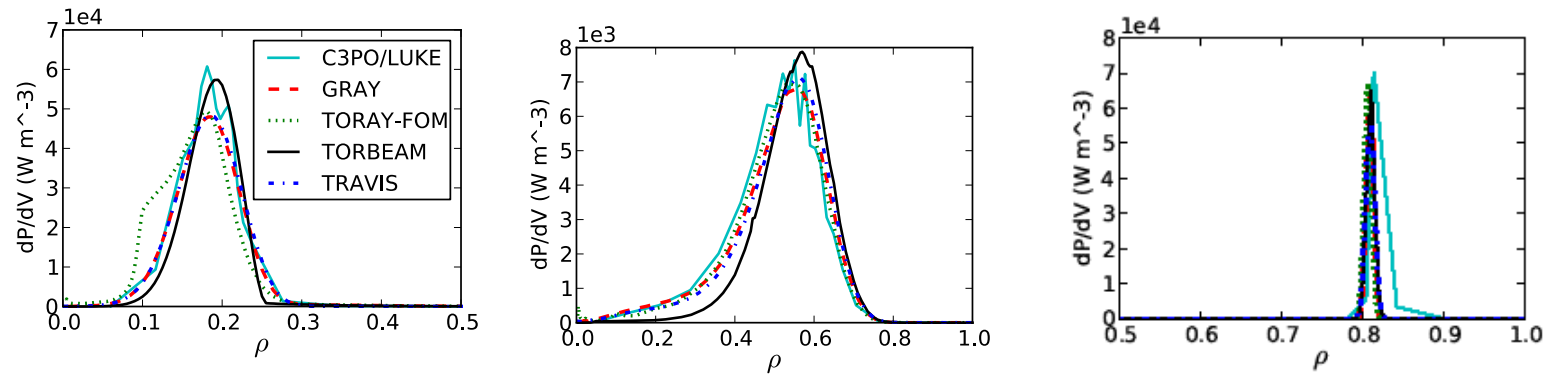

Figure 12 Power density profiles computed for the launching conditions of Table 1: EL25 (left), EL4O (center)[6 with kind permission of the European Physical Journal (EPJ)] and UL (right). 


\subsection{European Transport Simulator validation}

A rigorous benchmarking of the ETS against ASTRA[31] and CRONOS[40] integrated modelling transport codes was performed by using the parameters of JET hybrid discharge \#77922 with current overshoot, $\mathrm{B}_{\text {tor }}=2.3 \mathrm{~T}, \mathrm{I}_{\mathrm{pl}}=1.7 \mathrm{MA}$, high triangularity $(0.38), 18 \mathrm{MW}$ of NBI, $\mathrm{n}_{\mathrm{l}}=4.8 \cdot 10^{19} \mathrm{~m}^{-3}, \beta_{\mathrm{N}}=2.8$. The equilibrium was reconstructed in CRONOS and ASTRA with the solvers available within those, respectively HELENA [12] and the three moments equilibrium module EMEQ [35]; the latter module was also implemented in the ETS. It is worth to mention that the flexibility of the ETS advantageously allows for an easy integration of additional equilibrium codes other than those it already supports. Evidently ideally a rigorous benchmark should have been required all codes to use the same equilibrium reconstruction.

Self-consistent evolution of electron and ion temperatures, current diffusion and equilibrium was simulated. Spitzer resistivity was used for the current transport and the heat transport coefficients were provided by a Bohm - gyro-Bohm transport model. Neoclassical heat transport was not included. The simulations were performed with a fixed electron density profile measured at 7.7s of shot \#77922. Gaussian heating and current drive profiles (centred at $\rho=0$, half-width $\Delta \rho=0.3$ ), with a total heating power $\mathrm{P}_{\text {tot }}=18 \mathrm{MW}$, distributed $70 / 30$ between ions and electrons, were used in all codes. A beamdriven current $\mathrm{I}_{\mathrm{ni}}=0.12 \mathrm{MA}$ was imposed in all simulations while the bootstrap current was neglected. With these assumptions, the simulations were performed for 40 s reaching a steady-state solution.

A satisfactory agreement was obtained on the temperatures and q-profile simulated by the three codes as well as on the computed thermal diffusivities (Figure 13) [6]. The slight differences in profiles can be attributed to the different equilibrium solvers used within the compared integrated modelling transport codes.
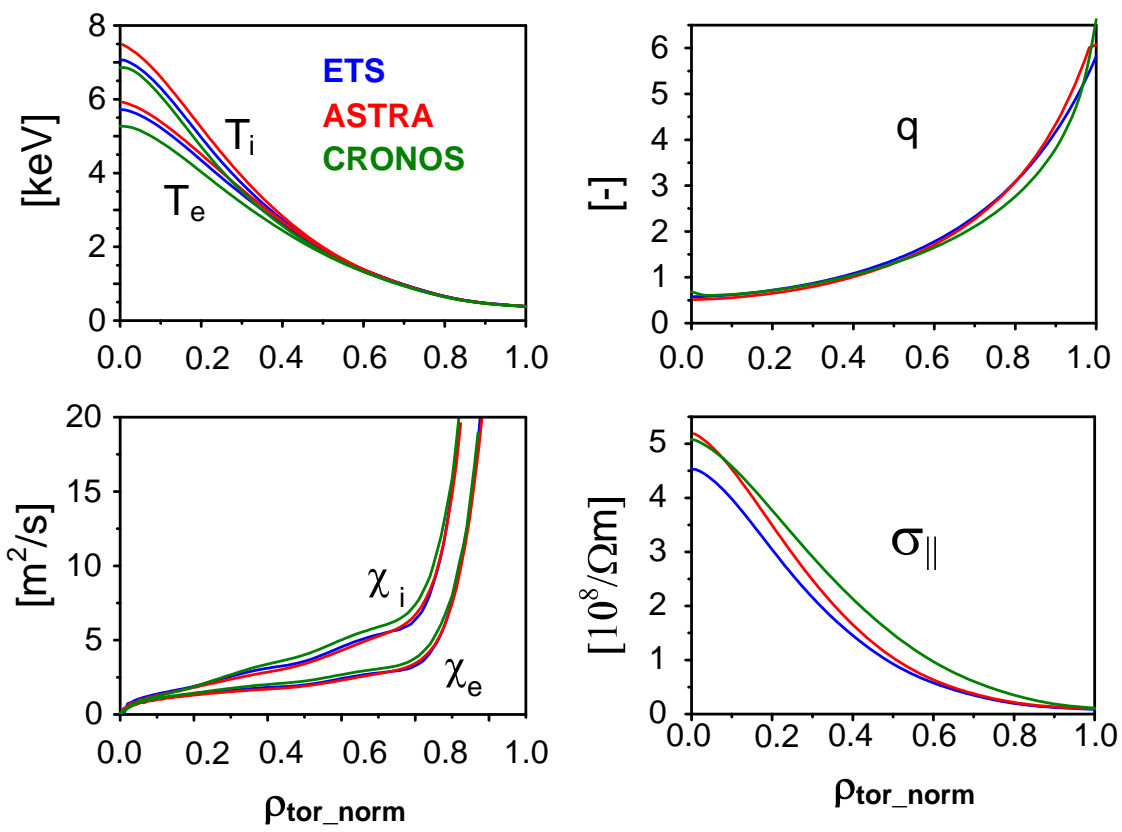

Figure 13 Benchmarking between ETS (blue), ASTRA(red) and CRONOS(green) integrated modelling transport codes for the conditions of JET hybrid discharge \#77922. Profiles of ion and electron temperature, safety factor, ion and electron heat diffusivity and parallel resistivity are plotted in the steady-state, after 40s of evolution.

\section{Tightly coupled workflows developed by ITM-TF}

\subsection{Core-edge coupling}

Coupling codes, besides the complexity of dealing with separate codes eventually presenting mixedlanguage programming, which is indeed overcome by the ITM-TF approach, introduces a number of 
issues to be dealt with: disparity in time-scales, different physics assumptions and scheduling the interaction between the coupled codes.

The core-edge coupled system does introduce a disparity in time-scales, with a characteristic timescale for the core being an energy confinement time or longer (seconds), whereas the Scrape-Off Layer (SOL) typically has a time-scale of milliseconds with some phenomena being even faster. Another disparity is the computational complexity: transport solvers for the core are typically 1D (radial) codes solving a set of reaction-convection-diffusion equations evolving the density, toroidal momentum and energies for the species considered; edge transport solvers are typically a 2D (radial and poloidal with toroidal symmetry assumed) or 3D code solving for the density, parallel momentum and energies for the species considered and are thus considerably more expensive computationally. Moreover impurities in the core are often split off from the main ion species and only the density equations are solved for the various impurity charge states. The coupling effort is significantly simplified in the case one is interested in finding a consistent steady state solution between the core and edge codes, which is the problem addressed here.

Three approaches for core-edge coupling can be used, as described in [41]: mediated, where the edge codes are used to provide boundary conditions for the core codes on the basis of fitting coefficients to the results of a number of edge runs; direct where the edge and core codes are directly coupled; and avoided where the edge code is extended all the way to the centre of the plasma. There have been several previous independent core-edge coupling projects: [42] describes the coupling of the core code Corsica to the edge code UEDGE; [44] describes the coupling of JETTO, EDGE2D and SANCO. A very similar approach describing the coupling of SOLPS and ASTRA is described in [45][46]. An alternative approach is that described in [41] where the coupling issue was avoided by extending the SOLPS grid to the centre of the machine. In [47] scaling laws were derived on the basis of SOLPS simulations and then used for core simulations.

Here we present the direct coupling of an edge and a core transport code via a Fortran workflow using the ITM-TF infrastructure (ie CPOs) for the particular case of steady state and multiple impurities [7]. The edge 2D transport code (SOLPS) [43] was coupled with the 1D core main plasma transport code ETS [4] including a core impurity transport code, developed within the framework of the ITM-TF. In this work a Fortran version of the ETS workflow was used, including the equilibrium code HELENA[12] and simple models for particle and energy sources as well as transport coefficients. ASDEX Upgrade shot \#17151 equilibrium at 2.5s was imported into equilibrium and limiter CPOs. These CPOs enter the HELENA code providing equilibrium to the core transport code and were used to create the SOLPS grid (Figure 14 left).
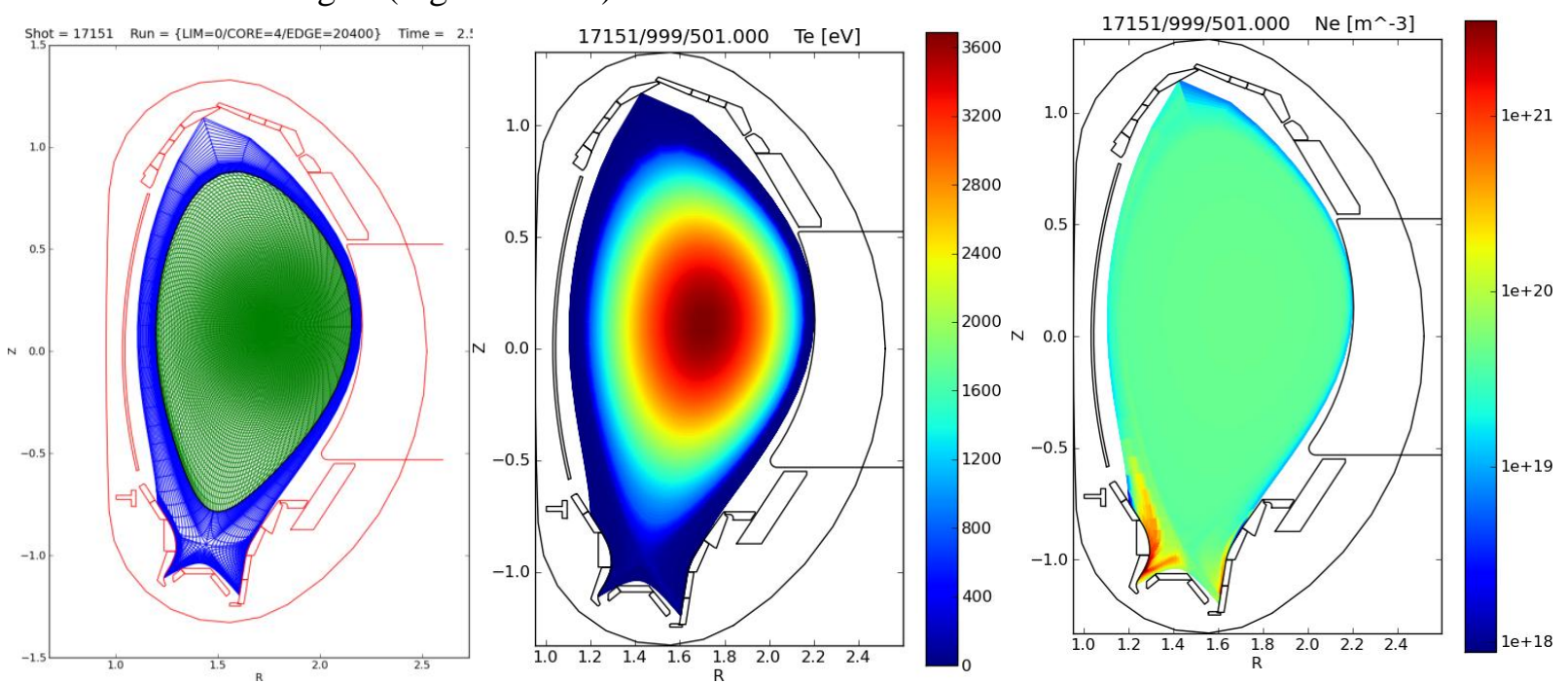

Figure 14 Left, the combined core and edge grids for ASDEX Upgrade shot 17151. The boundary surface separating the calculation domains between the core and edge codes is clearly visible. $T_{e}$ (center) and $n_{e}$ (right) for the final state of the $\mathrm{D}+\mathrm{He}+\mathrm{C}+\mathrm{Ar}+\mathrm{Ne}$ case. [7] All plot data are derived from CPOs. 
The location of the transition surface between the core and edge code was chosen at $95 \%$ of the normalized poloidal flux for the case shown below, as this is the usual stopping point for 1D core transport and equilibrium codes, making comparisons to existing results easier. Standalone SOLPS runs, pertaining to the demonstration case chosen here, showed the poloidal asymmetries to have averaged out at this depth. More generally, at least for H-mode cases, any poloidal asymmetries introduced by the edge physics do not penetrate past the pedestal, as demonstrated by a comparison study between SOLPS and ONETWO codes [48].

The two codes, ETS and SOLPS, were then called alternately and individually run until converged, with information about the boundary conditions transferred from one to the other, until convergence of the workflow is obtained.

For the most complicated test case, SOLPS treated all of the charge states of D, He, C, Ar and $\mathrm{Ne}$ (including the neutrals), a total of 42 . The ETS treated $\mathrm{D}^{+}$and $\mathrm{He}^{+2}$ as main ions, and the core impurity code treated the individual charge states of $\mathrm{C}, \mathrm{Ar}$ and $\mathrm{Ne}$. The core codes did not, in this case, treat the neutrals. Electron and ion energy fluxes as well as $\mathrm{D}+$ and $\mathrm{He}^{+2}$ particle fluxes are passed from the core to the edge code. Values of density and ion temperature on the boundary are passed from SOLPS to the ETS and densities of $\mathrm{C}, \mathrm{Ar}$ and Ne charge states to the core impurity code. SOLPS used a zeroflux boundary condition for neutrals, all of the charge states of $\mathrm{C}, \mathrm{Ar}, \mathrm{Ne}$ and for $\mathrm{He}^{+1}$. The fluxes are implemented in the edge code via a feedback loop which varies a constant density on the boundary so that the desired flux is obtained; this avoids the problem of having a poloidal variation of the density on the boundary which would then need to be averaged in some way before being passed back to the core code (if the flux boundary condition is implemented directly using a prescription of equal flux per unit area, a strong poloidal variation for some of the impurity densities can be found).

Convergence was obtained with 5 iterations as it is shown in Figure 15. To illustrate the convergence Figure 16 shows the time evolution of the electron and ion temperature profiles at the outer midplane. The results for the steady state electron temperature and density are shown in Figure 14 (centre, right); densities for $\mathrm{C}, \mathrm{Ne}$ and Ar charge states in Figure 17.
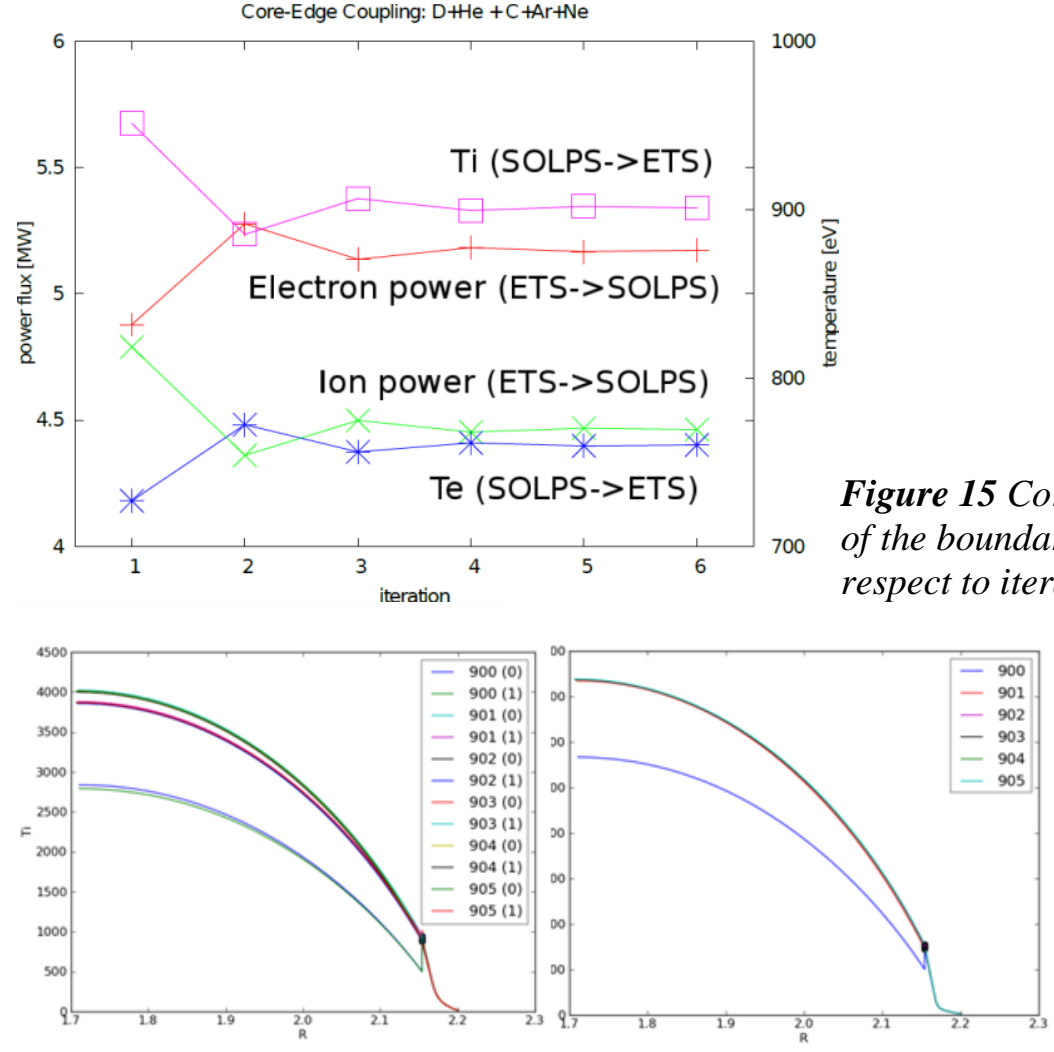

Figure 15 Core-edge workflow convergence of the boundary powers and temperatures with respect to iteration number.

Figure 16 Time evolution of the ion and electron temperature (left, centre, respectively) and electron density (right) profiles at the outer midplane.

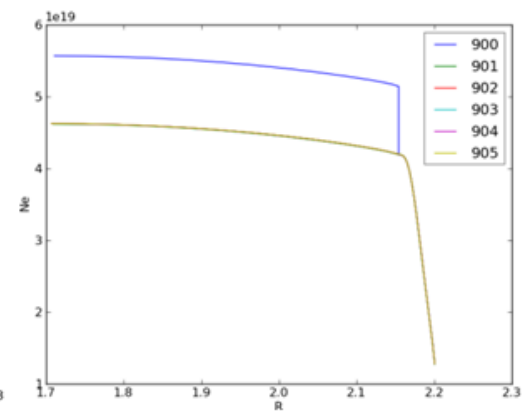




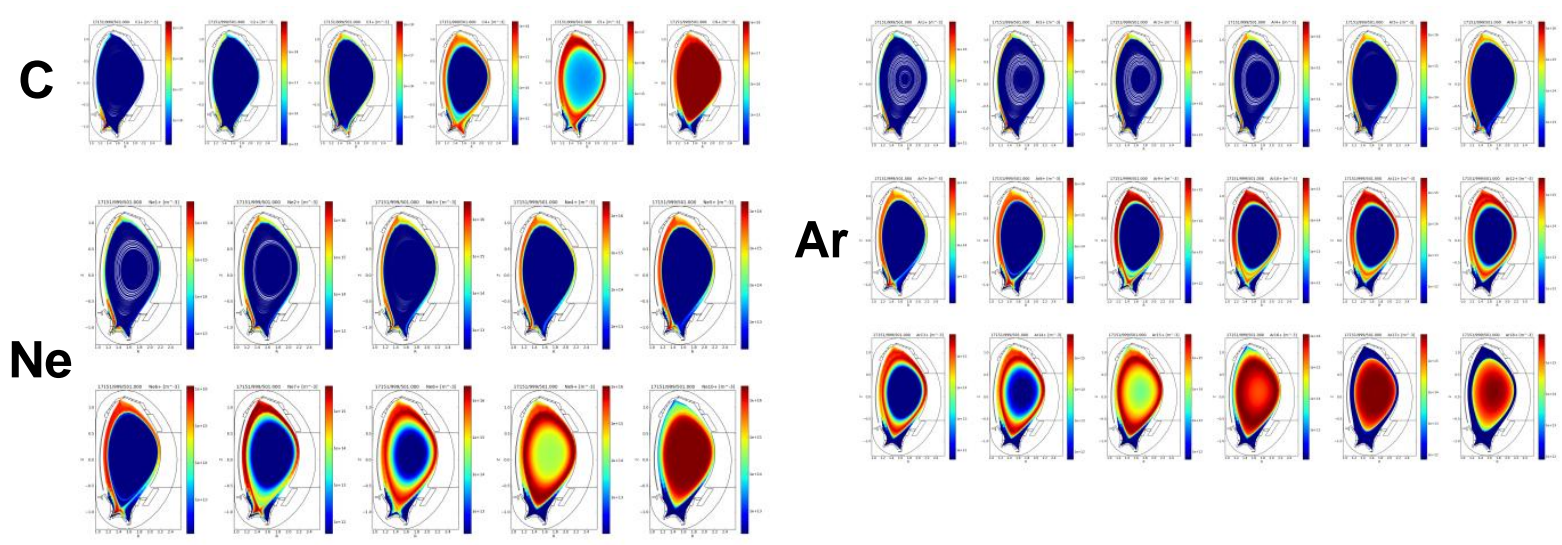

Figure 17 Density plots in the steady state for all the charges states of $\mathrm{C} \mathrm{Ne}$ (left, top bottom) and Ar (right)[7]

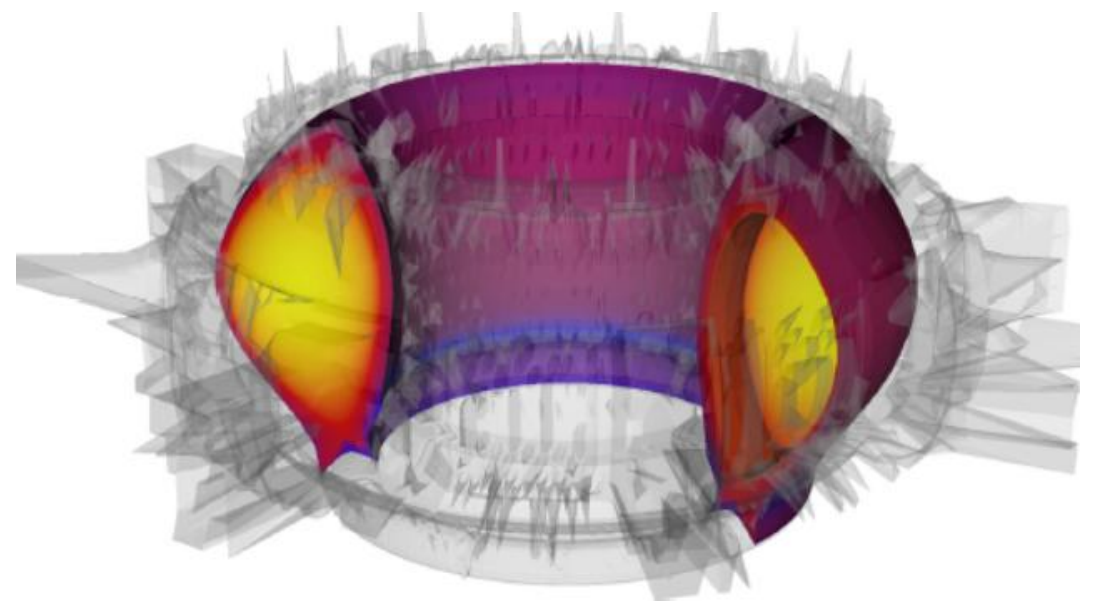

\begin{abstract}
Figure 18 Visualization of the core-edge coupled simulation results: Te calculated in the core with the ETS, in the edge with SOLPS, within the $3 D$ defeatured first wall of ASDEX Upgrade obtained using a raytracing rasterization and smoothing [49]. All data is stored in CPOs and plot with VisIT.
\end{abstract}

Figure 18 shows a visualisation of the core plasma temperature (simulated in 1D by the ETS core transport solver), the edge plasma temperature (simulated in 2D by SOLPS) together with the 3D wall. Recently SOLPS has been modified so that it can accommodate time-dependent boundary conditions . The coupling has thus been automated as follows: the ETS Fortran workflow calls SOLPS just after the convergence loop, SOLPS receives as input the necessary boundary conditions from the core CPOs, runs for one or more time-steps and calculates new core CPOs with new boundary conditions based on the edge results, then the ETS continues with a new time-step. The advantage of the new automated coupling scheme goes beyond just speeding up the calculation. The initial approach relied on a manual coupling which required the user not to make mistakes in the coupling procedure, and was also limited to steady-state scenarios. For impurities, there was also a limitation in that only cases with net zero flux could be implemented, and this was then done by charge state rather than the more physically correct summation over charge states. The new approach does not require manual intervention during the run, is not limited to steady state simulations, and removes the issue related to zero flux boundary conditions for impurities at the coupling interface.

\subsection{Transport simulations including NTMs}

A module which simulates the time behaviour of Neoclassical Tearing Modes (NTM) [50] can also be integrated in the ETS workflows. Here we present a demonstration of the ETS workflow including the NTM module reproducing the effect of NTMs on transport evolution.

NTMs are resistive instabilities breaking the flux surfaces into magnetic islands at the rational surfaces $\mathrm{q}=\mathrm{m} / \mathrm{n}$ (i.e. located at radius $\mathrm{r}_{\mathrm{s}}$ ). The modes are destabilized by a loss of bootstrap current proportional to the plasma pressure. The simulated modes grow starting from a specified onset time, up to the saturated state. Their growth affects the local electron and ion temperature and density by changing the perpendicular transport coefficients around the mode location. The transport is modified by the NTM 
module, which adds a Gaussian perturbation of given amplitude and width to the unperturbed transport coefficients [51]. The width is calculated self-consistently by solving the modified Rutherford equation at each simulation time-step, with parameters as in [52], evaluated in toroidal geometry, except assuming $\Delta^{\prime}=-\mathrm{m} / \mathrm{r}_{\mathrm{s}}$ (effective $\Delta^{\prime}$ in case of large island, $\Delta^{\prime}$ being the usual tearing parameter due to the perturbation of the equilibrium current). This approach enables the reproduction of density and temperature profiles very close to the experimental ones.

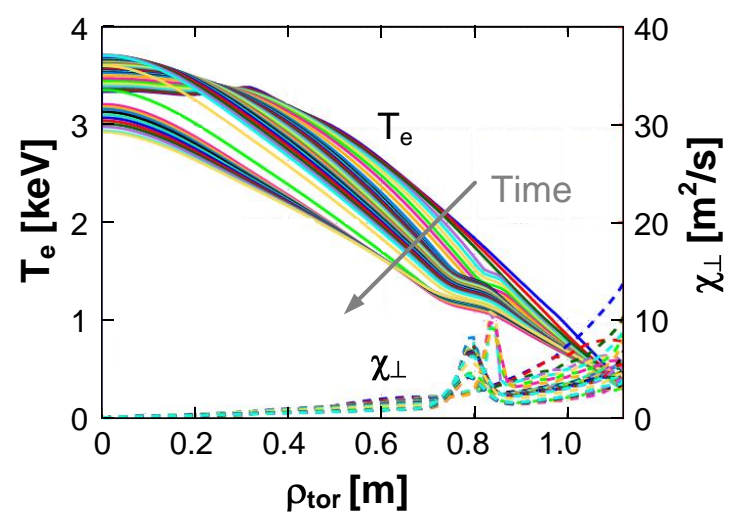

Figure 19 Modification of the heat transport coefficient by NTMs, assumed to be located at $\rho_{t o r} \sim 0.8$, and its effect on the electron temperature profile.

Figure 19 presents the temporal evolution of the electron temperature and total perpendicular heat diffusivity profiles, during an ETS-NTM simulation performed for typical JET H-mode plasma conditions. The effect on these profiles of an $\mathrm{m} / \mathrm{n}=2 / 1$ magnetic island, assumed to be located at $\rho_{\text {tor }}$ $\sim 0.8$ is shown. NCLASS [53] is used within the ETS [33] to calculate the resistivity and bootstrap current. The equilibrium is evolving and parameters in the modified Rutherford equation are recalculated according to [52]. The q profile is thus slightly evolving which leads to a radial displacement of the $\mathrm{q}=\mathrm{m} / \mathrm{n}$ surface and therefore a change of location of the increased transport due to the island (peak on $\chi$ ). The increase of radial transport due to the presence of the magnetic island leads to a flattening of the temperature profile around the $2 / 1$ surface. The mode is predicted to grow up to a saturated island of $8 \mathrm{~cm}$ width on a resistive time scale of about $150 \mathrm{~ms}$; this leads to a $16 \%$ drop in the stored energy. Validation against experimental data will be the next step and requires first a validation of the transport model.

\subsection{Free boundary equilibrium coupled to transport}

A key feature for a tokamak simulator is the inclusion of the Poloidal Field (PF) system, i.e. the PF coils and their power supplies as well as passive conducting structures. This allows including important operational limits and real-time magnetic control issues in the design of scenarios [54]. The ETS now has such a capability thanks to the inclusion of a Free-Boundary Equilibrium (FBE) solver, at present, either CEDRES++[55] or FREEBIE [56][57]. The circuit equations for the PF coils and passive structures are embedded in the FBE code. A switch in the ETS workflow allows to select one of these solvers in place of a fixed-boundary one. Coupling a FBE code to a 1D transport solver is not trivial [58],[55] therefore the coupling algorithm is detailed in the following.

In order to ensure consistency between the equilibrium and the profiles, the FBE-transport coupling scheme relies on a convergence loop performed at each time step, which is represented in Figure 20.

A time step starts with one transport step $t \rightarrow t+d t$. In addition to the coreprof_CPO which contains the profiles at time $t$, the transport solver needs as an input an equilibrium_CPO, for two reasons: the transport equations involve metric coefficients which depend on the equilibrium (e.g. such as the flux surface average of 1/R) and the Flux Diffusion Equation (FDE) needs a Boundary Condition (BC) at the edge, which has to be provided by the equilibrium. This BC is a central point of the FBE-transport coupling. It has to guarantee in particular the consistency of the poloidal flux $\Psi$, which is evolved both by the FBE solver $\left(\Psi_{\text {eq }}\right)$ and the transport solver $\left(\Psi_{\text {tr }}\right)$. The natural choice for the BC of the FDE, 
$\Psi_{\mathrm{tr}, \mathrm{x}=1}=\Psi_{\mathrm{eq}, \mathrm{xb}}$, where $\Psi_{\mathrm{eq}, \mathrm{xb}}$ is the poloidal flux at the plasma boundary provided by the equilibrium solver at the previous iteration of the convergence loop (or at the previous time step for the first iteration) and $\mathrm{x}$ is the normalized square root of the toroidal flux, tends to generate unphysical current

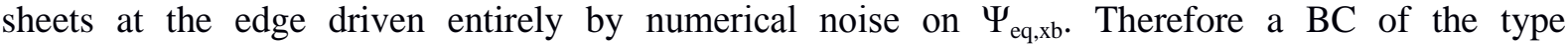
$\left.\frac{d \psi}{d x}\right|_{x=1}=\frac{-2 \pi \mu_{0} I_{p}^{*}}{C_{2}(x=1)}$

is used, where $\mathrm{C}_{2}$ is a metric coefficient (see [54]). In order to guarantee consistency the following expression is used

$$
I_{p}^{*}=I_{p, e q}\left[1+\tanh \left(\frac{\psi_{t r, x b}-\psi_{e q, x b}}{\psi_{e q, x 0}-\psi_{e q, x b}}\right)\right] \text { Equation } 1
$$

where subscripts $x 0$ and $x b$ indicate the magnetic axis and the plasma boundary respectively.

This expression is based on the following considerations. A correction term $\Delta I_{p}$ is added $I_{p}{ }^{*}=I_{p, e q}+\Delta I_{p}$ ( $I_{p, e q}$ indicating the plasma current found by the FBE solver) aiming at ensuring the consistency between $\Psi_{e q}$ and $\Psi_{t r}$, which otherwise diverge one from the other. As a measure of the distance between those $\Delta \Psi_{x b} \equiv \Psi_{t r, x b}-\Psi_{e q, x b}$ is used; therefore, $\Delta I_{p}=\Delta \Psi_{x b} / L_{p}$, where $L_{p}$ has the dimension of an inductance and is chosen as $L_{p}=\left(\Psi_{e q, x 0^{-}} \Psi_{e q, x b}\right) / I_{p, e q}$. Finally a tanh function is used in order to provide a saturation of the correction term in case the initial difference $\Delta \Psi_{x b}$ would be large. Evidently, at the end of the convergence process, $\Delta \Psi_{x b}$ is small so that the tanh does not make a difference.

After the transport time step, in order to prepare the input data for the FBE time step, an intermediate step is necessary to calculate the $p^{\prime}\left(\Psi_{n}\right)$ and $f f^{\prime}\left(\Psi_{n}\right)$ profiles from the new $p$ and $\Psi$ profiles and the metric coefficients. $\Psi_{n}$ is the normalized $\Psi$ equal to 0 on the magnetic axis and 1 at the plasma boundary, $f=R B_{\varphi}$ is the diamagnetic function and the 'denotes the derivative with respect to $\Psi$. First, $p^{\prime}$ is calculated as $p^{\prime}=\frac{d p / d x}{d \psi / d x}, f f f^{\prime}$ is then obtained from the averaged Grad-Shafranov equation [54].

An FBE step $t \rightarrow t+d t$ can then be made, using as inputs the $p$ ' and $f f$ ' profiles as well as the voltages in the PF coils (at present those are prescribed but will eventually be provided by a magnetic controller). The FBE time step calculates a new equilibrium at $t+d t$, including new metrics and a new $I_{p}$. These are then injected back into the transport solver, and the whole process is repeated.

The convergence criterion ensures that the difference in the averaged current density $j_{a v} \equiv\left\langle j_{\varphi} / R\right\rangle /\langle 1 / R\rangle$ (where brackets denote a flux surface average) and $\Psi_{e q, x b}$ between two iterations is smaller than a given tolerance.

It has to be noted that this algorithm works for both limited and diverted plasmas. As a demonstration of the coupled FBE-ETS workflow we present here a simulation of a Vertical Displacement Event (VDE) in ITER. The initial plasma has $I_{p}=11.8 \mathrm{MA}$, an elongation $\kappa=1.49$, and is limited on the high field side (HFS). PF voltages are set to 0 . Figure 21, shows the behaviour of the magnetic axis vertical position $Z_{\mathrm{a}}$. As expected, it has an exponential behaviour. The time constant is $\tau_{\mathrm{VDE}}=102 \mathrm{~ms}$, which is typical of ITER [54]. In Figure 22, the toroidal current density $j_{\varphi}(R, Z)$ is shown at two times in the simulation: 108.50 and $108.82 \mathrm{~s}$ (the simulation starts arbitrarily at $\mathrm{t}=108 \mathrm{~s}$ as shown in Figure 21). It can be seen that as the plasma moves down, negative currents are induced in the passive structures (as one may expect), in particular the triangular support (small oblique plate on the LFS) and, to a smaller extent, the divertor inboard rail (small vertical plate on the HFS) and the lower part of the vacuum vessel shells (mostly the inner one). Interestingly, a positive current sheet grows at the edge of the plasma towards the end of the simulation. This has to be analysed in detail but it is likely a consequence of the growth of the negative currents in the passive structures. We note that the global current diffusion $(\mathrm{L} / \mathrm{R})$ time of the plasma here is of several thousands of seconds whereas the local time at the plasma edge is of the order of few seconds, on the spatial scale of the observed current sheet, consistently with the simulation. As for the previous section case, the plasma resistivity is calculated with NCLASS within the ETS. There is no bootstrap current spike at the edge however, because this plasma has L-mode-like profiles. 


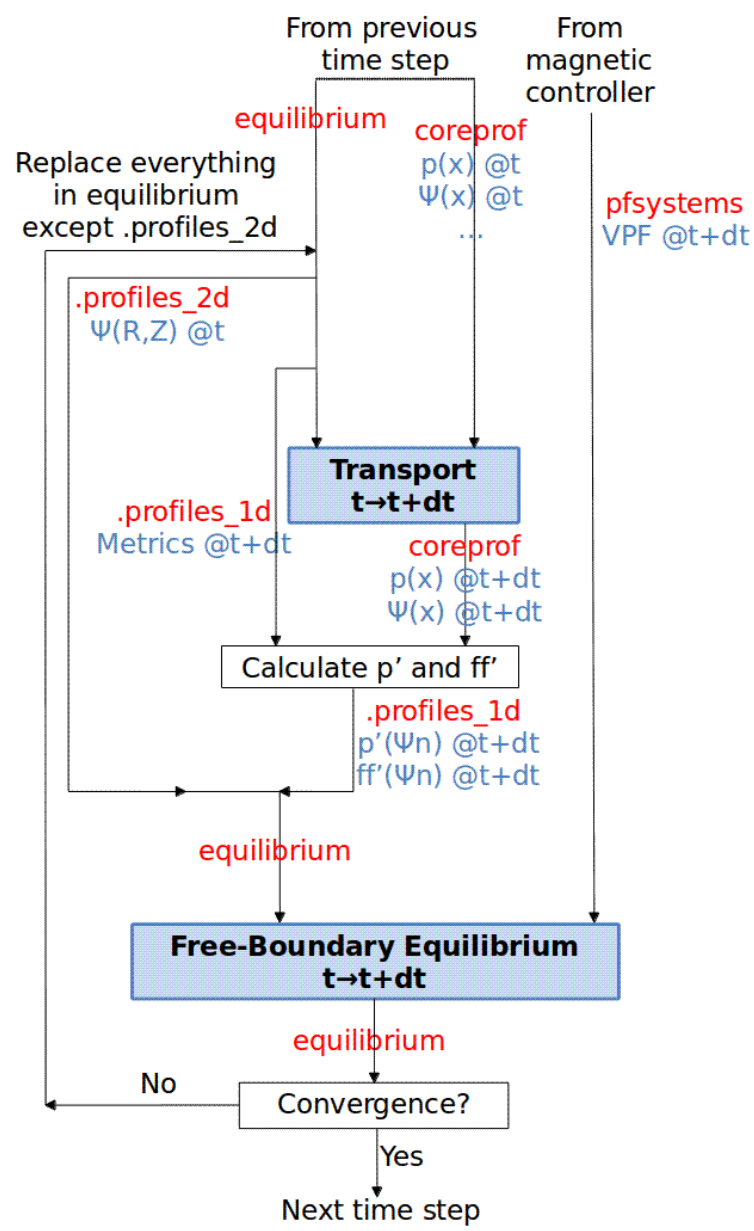

Figure 20 The convergence loop performed at each time step in the coupled FBE ETS workflow. The labels next to the arrows comprise, in red, the names of the transferred CPOs or CPO fields and in blue, the names of the main variables of interest which are contained within them.

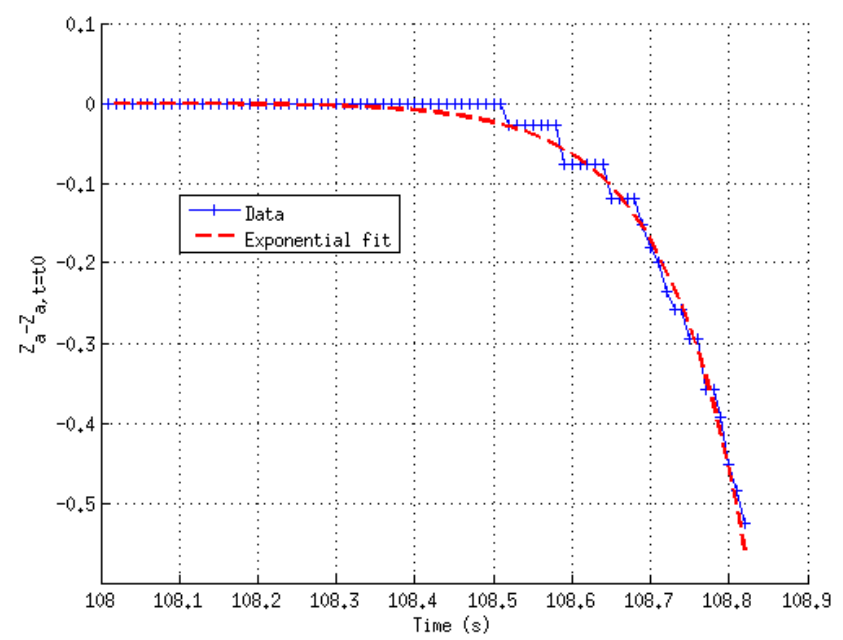

Figure 21 Evolution of the magnetic axis vertical position with respect to its initial value. 

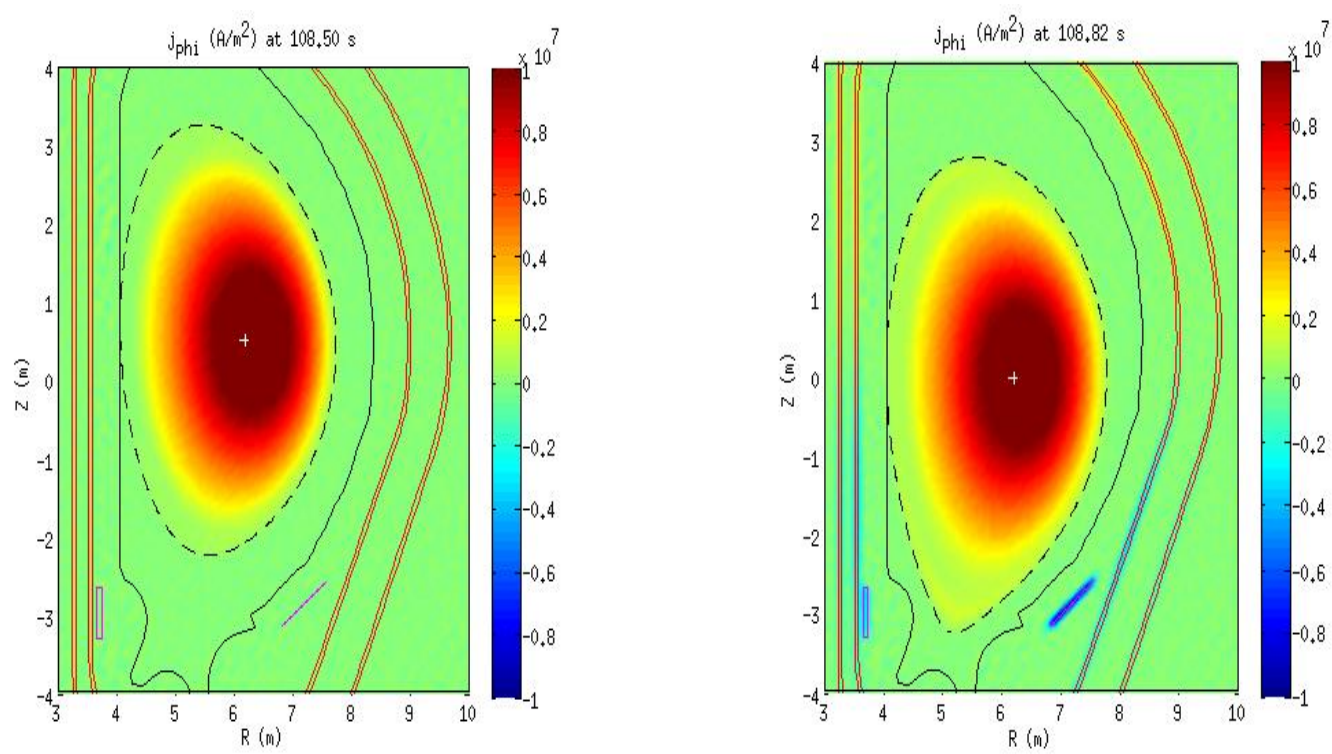

Figure 22 Snapshots of the toroidal current density $j_{\varphi}$ at $t=108.50 \mathrm{~s}$ (left) and $108.82 \mathrm{~s}$ (right). The white cross indicates the magnetic axis, the black dashed line the separatrix, the black full line the first wall, and the red and magenta lines the vacuum vessel shells and passive structures respectively (divertor inboard rail and triangular support).

Subsequent to this first demonstration of the FBE-ETS workflow, a cross-benchmarking of the FBE codes (CEDRES++ and FREEBIE) within the above detailed workflow has been started ; possibly, optimizations of the algorithm and benchmarks with existing similar efforts are foreseen as well.

The main following step is the implementation of a feedback controller to allow for scenario simulations. Preliminary work has already been performed in this direction with the inclusion of a controller actor produced from a TCV Simulink controller in a CEDRES++ workflow (without coupling to the ETS, hence using prescribed $p$ ' and $f f$ ' profiles), which allowed reproducing a "yo-yo" $\mathrm{TCV}$ discharge, i.e. the plasma is moved up and down the vessel by the magnetic controller.

\section{Synthetic diagnostics integration}

The ongoing efforts on synthetic diagnostic integration in the ITM-TF platform focus on reflectometry, neutron and NPA diagnostics and spectral MSE.

A full-wave 3D code (ERC3d) valid for both $\mathrm{O}$ and $\mathrm{X}$-mode polarizations has been developed, ported and tested on the ITM platform and work is under way to enhance the kernel to cope with high levels of turbulence and high injection angles (Doppler reflectometry operation). A generic framework for neutron synthetic diagnostics has been integrated which is composed of three different modules: calculation of the effective solid angle of the detector from small plasma volumes (LINE21 code); a Directional RElativistic Spectrum Simulator (DRESS) to derive the energy spectra and source rates of particles created in fusion reactions emitted in a specified direction and a diagnostic response function. Integration of JET neutron camera setup is ongoing. The integration of NPA diagnostics in the ITM platform was also carried out using modules of the ASCOT code package [59] and calculating the fraction of the tokamak chamber and born neutrals (with given pitch velocity) that are within the sight of the NPA collimator. A spectral MSE forward model [60] that calculates the emissivity for each MSE channel and the resultant radiance Balmer-alpha MSE spectra as well as the charge exchange of the plasma with the beam has been integrated. Full, half and third beam energy components are considered and a collisional-radiative beam-plasma model is used to determine the coupled densities of charged states along the diagnostic neutral beam path. Preliminary results on the MSE synthetic diagnostic validation on ASDEX Upgrade data (shot \#26320) are presented in Figure 23, showing the simulated and experimental emissivities. 
Figure 23 MSE emissivity wavelength spectra for ASDEX Upgrade shot \#26320. The contribution from half and third beam energy components, beam divergence and unshifted $D \alpha$ emission are shown.

An offset of $\sim 1000$ counts is added to the MSE $+C X$ synthetic counts to account for the characteristic background level of the measured signal by the $C C D$.

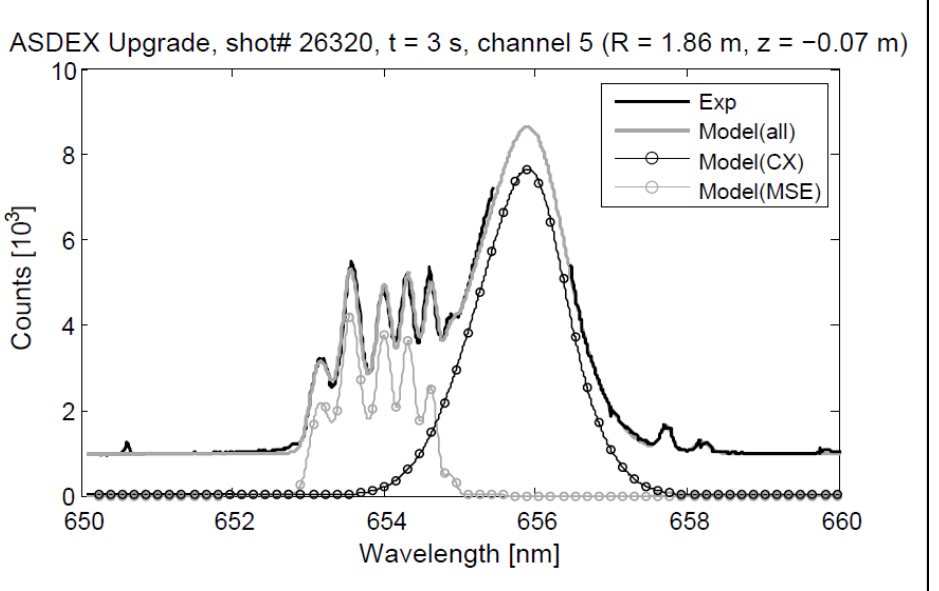

\section{Conclusions}

The EU ITM-TF standardized, modular and flexible integrated modelling framework allows building complex workflows for physics application and is a valuable environment to benchmark codes describing similar physics processes with different model sophistication, by interchanging those as modules within the same workflow. Besides, both the orchestration engine Kepler, and the ITM-TF developments performed in collaboration with the FP7 EUFORIA project [16] and the HLST $\ddagger$, allow to run workflows or only part of those (the main workflow residing on the central ITM Gateway cluster) on GRID or HPC-FF, thus rendering possible highly computationally demanding calculations. The first application of the ITM-TF simulation chain coupling equilibrium reconstruction, refinement and linear MHD stability modules addressed edge stability of ASDEX Upgrade ELMy H-Mode and ITER hybrid scenario. Turbulence code interpretative runs starting from given experimental profiles of a JET hybrid discharge, challenging case near to stability threshold conditions, were performed with two different electromagnetic codes, a gyrofluid and a gyrokinetic one, within an ITM workflow. Only one radial position ( $\mathrm{ra}=0.6$ in normalised radius) was found unstable in the gyrokinetic run, highlighting trapped-electron assisted ITG turbulence characteristics. A benchmark among EC beam/ray-tracing codes for a standard inductive H-mode ITER scenario for three different launching conditions, showed good agreement of the five EU codes even in the more demanding test cases, like central ECCD at high temperature, and beam focused close to the resonance region. Benchmarking of the European Transport Simulator (ETS) against ASTRA and CRONOS transport codes, on a JET discharge, showed very good agreement among the simulated quantities, laying the foundations for its usage for both predictive and interpretative runs on present devices and ITER.

Some selected examples of ITM scientific workflow applications have also been outlined. Automated direct coupling of a core and edge transport code was demonstrated for the particular case of steady state and multiple impurities. The effect of NTMs on heat transport coefficients and temperature profiles was reproduced via a dedicated NTM module incorporated in the ETS. Coupling of the ETS to a free boundary equilibrium solver was tested on a vertical displacement event (VDE) for an ITER scenario. Finally, ongoing efforts on the integration and testing of synthetic diagnostics in the ITM-TF platform have been reported, namely, the validation of spectral MSE forward model on ASDEX Upgrade data.

\section{Acknowledgments}

\footnotetext{
$\$$ http://www.efda-hlst.eu/
} 
This work, supported by the European Communities under the contract of Association between EURATOM and CEA, CCFE, ENEA, FOM, IPP, IPPLM, IST, Swiss Confederation, VR, was carried out within the framework of the Task Force on Integrated Tokamak Modelling of the European Fusion Development Agreement. Part of the research leading to these results received funding from the European Community's Research Infrastructures initiative of the 7th Framework Programme FP7 (2007-2013) under grant agreement No 211804 (EUFORIA). The views and opinions expressed herein do not necessarily reflect those of the European Commission. Dr. X. Bonnin and O. Hoenen are gratefully acknowledged for their collaboration in the revision of the paper.

\section{Appendix: List of ITM-TF contributors}

M. Airila ${ }^{1}$, S. Äkäslompolo ${ }^{2}$, L. Allegretti ${ }^{3}$, L. Alves ${ }^{4}$, T. Aniel ${ }^{3}$, L. Appel ${ }^{5}$, J-F. Artaud ${ }^{3}$, O. Asunta ${ }^{2}$, C-V. Atanasiu 6 , F. Aumayr ${ }^{7}$, O. Barana ${ }^{3}$, M. Baruzzo ${ }^{8}$, V. Basiuk ${ }^{3}$, R. Bilato ${ }^{9}$, J. Bizarro ${ }^{4}$, E. Blanco ${ }^{21}$, J. Blum $^{10}$, T. Bolzonella ${ }^{8}$, X. Bonnin ${ }^{11}$, D. Borodin ${ }^{12}$, A. Bottino ${ }^{9}$, C. Boulbe ${ }^{10}$, S. Brémond ${ }^{3}$,

S. Briguglio ${ }^{13}$, Y. Buravand ${ }^{3}$, A. Cardinali ${ }^{13}$, C. Cianfarani ${ }^{13}$, J. Citrin ${ }^{14}$, R. Coelho ${ }^{4}$, D. Coster ${ }^{9}$, G. Csepany ${ }^{17}$, D. Dimopoulos ${ }^{18}$, A. Dinklage ${ }^{19}$, C. DiTroia ${ }^{13}$, C. Dritselis ${ }^{18}$, D. Dumitru ${ }^{6}$, R. Dumont ${ }^{3}$,

E. Fable ${ }^{9}$, G L Falchetto ${ }^{3}$, D. Farina ${ }^{20}$, B. Faugeras ${ }^{10}$, J. Ferreira ${ }^{4}$, L. Figini ${ }^{20}$, A. Figueiredo ${ }^{4}$,

G. Fogaccia ${ }^{13}$, V. Fusco ${ }^{13}$, K. Gal $^{41}$, J. Garcia ${ }^{3}$, P. Garcia-Muller ${ }^{21}$, L. Garzotti ${ }^{5}$, J. Geiger ${ }^{9}$,

E. Giovannozzi ${ }^{13}$, G. Giruzzi ${ }^{3}$, V. Goloborodko ${ }^{16}$, R. Goswami ${ }^{3}$, B. Guillerminet ${ }^{3}$, S. Hacquin ${ }^{3}$,

A. Hannan ${ }^{22}$, J. Heikkinen ${ }^{1}$, T. Hellsten ${ }^{22}$, S. Heuraux ${ }^{23}$, J. Hillairet ${ }^{3}$, E. Hirvijoki ${ }^{2}$, J. Hobirk ${ }^{9}$,

D. Hogeweij ${ }^{14}$, L. Höök ${ }^{22}$, P. Huynh ${ }^{3}$, K. Igenbergs ${ }^{24}$, F. Imbeaux ${ }^{3}$, H. Isliker ${ }^{15}$, I. Ivanova-Stanik ${ }^{25}$, S. Janhunen ${ }^{2}$, F. Jenko ${ }^{9}$, T. Johnson ${ }^{22}$, S. Kakarantzas ${ }^{18}$, N. Kanaris ${ }^{26}$, S. Kassinos ${ }^{26}$, A. Keim ${ }^{7}$, V. Kiptily ${ }^{5}$, A. Kirschner ${ }^{12}$, T. Kiviniemi ${ }^{2}$, H-J. Klingshirn ${ }^{9}$, F. Köchl ${ }^{7}$, Y. Kominis ${ }^{35}$, T. Korpilo ${ }^{2}$, L. Kos ${ }^{27}$, T. Koskela ${ }^{2}$, S. Kulovec ${ }^{27}$, C. Lechte ${ }^{28}$, E. Lerche ${ }^{29}$, X. Litaudon ${ }^{3}$, F. Liu ${ }^{3}$, Y. Liu ${ }^{5}$, J. Lönnroth ${ }^{5}$, T. Lunt ${ }^{9}$, G. Manduchi ${ }^{8}$, N. Marushchenko ${ }^{19}$, S. Mastrostefano ${ }^{30}$, R. Mayo ${ }^{21}$, J. Miettunen ${ }^{2}$, S. Moradi ${ }^{31}$, D. Moreau ${ }^{3}$, P. Moreau ${ }^{3}$, A. Morillas ${ }^{21}$, D. Muir ${ }^{5}$, Q. Mukhtar ${ }^{22}$, F. Nabais ${ }^{4}$, E. Nardon ${ }^{3}$, F. Nave ${ }^{4}$, A H. Nielsen ${ }^{32}$, R. Nouailletas ${ }^{3}$, S. Nowak ${ }^{20}$, M. O'Mullane, M. Ottaviani ${ }^{3}$, M. Owsiak ${ }^{33}$, V. Pais ${ }^{6}$, B. Palak ${ }^{33}$, A. Papadopoulos ${ }^{35}$, G. Papp ${ }^{17}$, N. Pelekasis ${ }^{18}$, Y.Peysson ${ }^{3}$, T. Pisokas ${ }^{15}$, M. Plociennik ${ }^{33}$, G. Pokol $^{17}$, E. Poli ${ }^{9}$, G. Poulipoulis ${ }^{36}$, I. Pusztai ${ }^{31}$, H. Radhakrishnan ${ }^{26}$, N. Ravenel ${ }^{3}$, H. Reimerdes ${ }^{37}$, D. Reiser ${ }^{12}$, M. Romanelli ${ }^{5}$, J. Rydén ${ }^{31}$,

A. Salmi ${ }^{1}$, T. Samaras ${ }^{35}$, O. Sauter ${ }^{36}$, P. Scheier ${ }^{37}$, K. Schmid ${ }^{9}$, M. Schneider ${ }^{3}$, K. Schöpf ${ }^{16}$,

B. D. Scott ${ }^{9}$, J. Signoret ${ }^{3}$, F. Silva ${ }^{4}$, S. Sipilä2 ${ }^{2}$, P. Siren ${ }^{1}$, A. Sirinelli ${ }^{5}$, A. Snicker ${ }^{2}$, R. Stankiewicz ${ }^{25}$, J. Storrs ${ }^{5}$, P. Strand ${ }^{38}$, E. Sundén ${ }^{39}$, T. Tala ${ }^{1}$, S. Tholerus ${ }^{22}$, G. Throumoulopoulos ${ }^{36}$, K. Tokesi ${ }^{40}$, C. Tsironis ${ }^{35}$, D. Tskhakaya ${ }^{16}$, O. Tudisco ${ }^{13}$, J. Urban $^{42}$, D. V.Eester ${ }^{29}$, L. Villard $^{36}$, F. Villone $^{30}$,

B. Viola ${ }^{13}$, S. Viorica ${ }^{6}$, G. Vlad $^{13}$, I. Voitsekhovitch ${ }^{5}$, E. Westerhof ${ }^{14}$, R. Wieggers ${ }^{14}$, M. Wischmeier ${ }^{9}$, D. Yadykin ${ }^{38}$, P. Zestanakis ${ }^{35}$, T. Zok ${ }^{33}$

1 VTT Technical Research Centre of Finland, Association Euratom-TEKES, P O Box 1000, 02037 VTT, Finland

2 Department of Applied Physics, Aalto University, Association Euratom-TEKES, PO Box 13500, FI-00076 AALTO, Finland

3 CEA, IRFM, F-13108 Saint-Paul-lez-Durance, France4 Associacão EURATOM/IST, Instituto de Plasmas e Fusão Nuclear, Instituto Superior Técnico, Universidade Técnica de Lisboa 1049-001 Lisboa, Portugal

5 EURATOM/CCFE Fusion Association, Culham Science Centre, Abingdon OX14 3DB UK

6 National Institute for Laser, Plasma and Radiation Physics, Association MEdC-EURATOM, Bucharest, Romania

7 Association EURATOM-ÖAW/ATI, Atominstitut, TU Wien, 1020 Vienna, Austria

8 Associazione EURATOM-ENEA sulla Fusione, Consorzio RFX, 29127 Padova, Italy

9 Max-Planck-Institut für Plasmaphysik, EURATOM-IPP Association, Garching, Germany

10 Univ. Nice Sophia Antipolis, Lab. JA Dieudonne, UMR 7351, F-06108 Nice 02, France

11 CNRS-LSPM, Université Paris XIII, F-93430 Villetaneuse, France

12 Institut für Energie und Klimaforschung Plasmaphysik, Forschungszentrum Jülich, Association Euratom-FZJ, Germany 
13 Associazione Euratom-ENEA sulla Fusione, C.R. ENEA-Frascati, Via E. Fermi 45, 00037 Frascati, Roma, Italy

14 FOM Institute DIFFER, Association EURATOM-FOM, Nieuwegein, Netherlands

15 Section of Astrophysics, Astronomy and Mechanics, Department of Physics, University of Thessaloniki, Association Euratom-Hellenic Republic, Thessaloniki, Greece

16 Association EURATOM-OAW, Institute for Theoretical Physics, University of Innsbruck, A-6020, Innsbruck, Austria

17 Department of Nuclear Techniques, Budapest University of Technology and Economics, Association EURATOM, H-1111 Budapest, Hungary

18 Department of Mechanical Engineering, University of Thessaly, Pedion Areos, Volos 38334, Greece - Association Euratom-Hellenic Republic

19 Max Planck Institut für Plasmaphysik, EURATOM Association, Greifswald, Germany

20 Istituto di Fisica del Plasma CNR, Euratom-ENEA-CNR Association for Fusion, Milan, Italy

21 Asociación EURATOM CIEMAT, Madrid 28034, Spain

22 Association EURATOM-VR, Fusion Plasma Physics, EES, KTH, SE-10037 Stockholm, Sweden

23 Université de Lorraine, IJL, UMR 7198, BP 70233, Vandoeuvre, F-54506 Cedex, France

24 Vienna Univ. Technol., Inst. Appl. Phys., A-1034 Vienna, Austria

25 Institute of Plasma Physics and Laser Microfusion, EURATOM Association,00-908 Warsaw, Poland

26 Computational Sciences Laboratory - UCY-CompSci, Department of Mechanical and Manufacturing Engineering, University of Cyprus, Nicosia, Cyprus

27 University of Ljubljana, Faculty of Mech. Eng., Askerceva 6, SI-1000 Ljubljana, Slovenia

28 Institute for Plasma Research, University of Stuttgart, 70569 Stuttgart, Germany

29 Ecole Royale Militaire - Koninklijke Militaire School, Laboratoire de Physique des Plasmas, B 1000 Brussels, Belgium

30 Associazione EURATOM/ENEA/CREATE, DIEI, Università degli Studi di Cassino e del Lazio Meridionale, Via Di Biasio 43, 03043 Cassino (FR), Italy

31 Nuclear Engineering, Department of Applied Physics, Chalmers University of Technology, Euratom-VR Association, SE-35296 Göteborg, Sweden

32 Association EURATOM-DTU, 3400 Roskilde, Denmark

33 Poznan Supercomputing and Networking Center (PSNC), IChB PAS, Noskowskiego 12/14, Poznan, Poland

34 School of Electrical and Computer Engineering, National Technical University of Athens, Association EURATOM-Hellenic Republic, Zografou, Athens 15773, Greece

35 University of Ioannina, Association Euratom - Hellenic Republic, Section of Theoretical Physics, Ioannina, Greece

36 Ecole Polytechnique Federale de Lausanne (EPFL), Centre de Recherches en Physique des Plasmas (CRPP), Association Euratom-Confederation Suisse, Lausanne, Switzerland

37 Institut für Ionenphysik und Angewandte Physik, Universität Innsbruck

38 Department of Earth and Space Sciences, Chalmers University of Technology, Euratom-VR Association, SE-352 96 Göteborg, Sweden

39 Uppsala University, VR-Euratom Association, Box 516, 75120 Uppsala, Sweden

40 Institute of Nuclear Research of the Hungarian Academy of Sciences (ATOMKI), 3401 Debrecen, Hungary

41 Institute for Particle and Nuclear Physics, Wigner Research Centre for Physics, Hungarian Academy of Sciences, EURATOM Association HAS, POB 49, H-1525, Budapest, Hungary

42 Association EURATOM/IPP.CR, IPP AS CR, Prague, Czech Republic

\section{References}

[1] Becoulet A, et al. 2007, Comput. Phys. Commun. 177(1-2):55 - 59.

[2] Strand P I, et al. 2010, Fusion Engineering and Design 85(3-4):383 - 387.

[3] Imbeaux F, et al, 2010, Comput. Phys. Commun. 181(6): 987 - 998.

[4] Coster D, Basiuk V, Pereverzev G, Kalupin D, Zagórksi R, et al., 2010, IEEE Transactions on Plasma Science 38, 2085. 
[5] Konz C, et al, 2011, "First physics applications of the Integrated Tokamak Modelling (ITM-TF) tools to the MHD stability analysis of experimental data and ITER scenarios", O2.103, 38th EPS Conference on Plasma Physics, Strasbourg. In Europhysics Conference Abstracts Vol. 35G, ISBN 2-914771-68-1, http://ocs.ciemat.es/EPS2011PAP/pdf/O2.103.pdf

[6] Figini L et al., 2012, "Benchmarking of electron cyclotron heating and current drive codes on ITER scenarios within the European Integrated Tokamak Modelling framework", EPJ Web of Conferences 32, 01011. EC-17- 17 ${ }^{\text {th }}$ Joint Workshop on Electron Cyclotron Emission and Electron Cyclotron Resonance Heating, http://dx.doi.org/10.1051/epjconf/20123201011

[7] Kalupin D, et al., 2011, "Verification and Validation of the European Transport Solver", P4.111, 38th EPS Conference on Plasma Physics, Strasbourg. In Europhysics Conference Abstracts Vol. 35G, ISBN 2-914771-68-1, http://ocs.ciemat.es/EPS2011PAP/pdf/P4.111.pdf

[8] Coster D P , Klingshirn H-J, et al., 2012, "Core-Edge Coupling: developments within the EFDA Task Force on Integrated Tokamak Modelling”, P1.073, 39th EPS Conference on Plasma Physics\& 16th Int. Congress on Plasma Physics, Stockholm. In Europhysics Conference Abstracts Vol. 36F, ISBN 2-914771-79-7, http://ocs.ciemat.es/epsicpp2012pap/pdf/P1.073.pdf

[9] Coelho R, et al., 2013, Fusion Science and Technology 63(1), 1-8.

[10] Zwingmann W, 2003, Nucl. Fusion 43, 842.

[11] Zwingmann W, et al., 2008, PLASMA 2007, AIP Conf. Proc. Vol. 993, 11.

[12] Huysmans G, Goedbloed J, and Kerner W, 1991, in CP90 Conf. on Comp. Physics Proc, page 371, World Scientific Publ. Co.

[13] Luetjens H, Bondeson A, Sauter O, 1996, Comput. Phys. Commun. 97, 219.

[14] Huysmans G T A, et al., 2001, Phys. Plasmas 8(10), 4292.

[15] Liu Y Q, Bondeson A, Fransson C M, Lennartson B, and Breitholtz C, 2000, Phys. Plasmas 7, 3681 .

[16] Zwingmann W, et al, 2010, "Validation Procedure of the Tokamak Equilibrium Reconstruction Code EQUAL with a Scientific Workflow System", P4-180, 37th EPS Conference on Plasma Physics, Dublin. In Europhysics Conference Abstracts Vol. 34A, ISBN 2-914771-62-2; http://ocs.ciemat.es/EPS2010PAP/pdf/P4.180.pdf

[17] Coster D P, Strand P, Contributors to the EUFORIA Project, 2010, "EUFORIA: Exploring EScience for Fusion", in Advances in Parallel Computing, 19, 520 - 529; DOI 10.3233/978-1-60750-530-3-520

[18] Goldston R J, McCune D C, et al, 1981 J. Comput. Phys. 43, 61.

[19] Scott B, 2005, Physics of Plasmas 12, 102307.

[20] Scott B, 2010, Physics of Plasmas 17, 102306.

[21] Scott B, 2000, Physics of Plasmas 7, 1845--1856.

[22] Scott B, 2001, Physics of Plasmas 8, 447--458.

[23] Naulin V, 2003, Physics of Plasmas 10 (10) , 4016--4028.

[24] Scott B, 2006, Plasma Phys. Contr. Fus. 48, B277-B293.

[25] Shestakov A I, et al., 2003, J. Computational Physics 185, 399.

[26] Candy J et al, 2009, Phys. Plasmas 16, 060704; http://dx.doi.org/10.1063/1.3167820

[27] Barnes M, et al, 2010, Physics of Plasmas 17, 056109.

[28] Kalupin D, et al. 2013, "Numerical analysis of JET discharges with the European Transport Simulator", Nucl. Fusion 53, 123007.

[29] Hoenen O, et al, 2013, "Designing and running turbulence transport simulations using a distributed multiscale computing approach", P4.155, 40th EPS Conference on Plasma Physics, Helsinki. http://ocs.ciemat.es/EPS2013PAP/pdf/P4.155.pdf

[30] Roberts S W, 1959, Technometrics 1, 239-250.

[31] Pereverzev G V and Corrigan G, 2008, Comput. Phys. Commun. 179 (8), 579.

[32] Basiuk V, Huynh Ph, et al., 2010, "European Transport Solver: first results, validation and benchmark", P 1.1009, 37th EPS Conference on Plasma Physics, Dublin. In Europhysics Conference Abstracts Vol. 34A, ISBN 2-914771-62-2; http://ocs.ciemat.es/EPS2010PAP/pdf/P1.1009.pdf

[33] Peysson Y, et al, 2012, Plasma Phys. Control. Fusion 54, 045003.

[34] Farina D, 2007, Fusion Sci. Technol. 52, 154. 
[35] Westerhof E, Implementation of TORAY at JET (Rijnhuizen Report, 1989) RR-89-183.

[36] Poli E, et al, 2001, Comp. Phys. Commun. 136, 90.

[37] Marushchenko N B, et al, 2007, J. Plasma Fusion Res. 2, S1000.

[38] Prater R, et al, Nucl. Fusion 48 (2008) 035006.

[39] Pereverzev G and Yushmanov P N, 2002, "ASTRA Automated System for Transport Analysis in a Tokamak", Max-Planck Institut fur Plasmaphysik, IPP 5/98.

[40] Artaud J-F, Basiuk V, Imbeaux F et al., 2010, Nuclear Fusion 50, 043001.

[41] Coster D P, 2009, Journal of Nuclear Materials 390-391,826.

[42] Tarditi A at al, 1996, Contributions to Plasma Physics 36 (2-3), 132-135, http://dx.doi.org/10.1002/ctpp.2150360207

[43] Schneider R, et al., 2006, Contrib. Plasma Phys. 46, 3, DOI 10.1002/ctpp.200610001

[44] Fichtmüller M, et al., 1998, Czechoslovak Journal of Physics 48, 25.

[45] Senichenkov I Y, et al, 2012, "Progress in ASTRA-B2SOLPS coupling for integrated tokamak modeling", P2.042, 39th EPS Conference on Plasma Physics, Stockholm. In Europhysics Conference Abstracts Vol. 36F, ISBN 2-914771-79-7, http://ocs.ciemat.es/epsicpp2012pap/pdf/P2.042.pdf

[46] Senichenkov I Y, et al, 2011, "Integrated modeling of H mode plasma in ASDEX Upgrade and Globus-M", P5.115, 38th EPS Conference on Plasma Physics, Strasbourg. In Europhysics Conference Abstracts Vol. 35G ISBN 2-914771-68-1, http://ocs.ciemat.es/EPS2011PAP/pdf/P5.115.pdf

[47] Kukushkin A S, et al, 2003, Nucl. Fusion 43, 716, doi:10.1088/0029-5515/43/8/312

[48] Owen L W, et al, 2010, Nuclear Fusion 50 (6), 064017.

[49] Äkäslompolo S, et al., 2012, "Preparing tokamak 3D wall and magnetic data for particle tracing simulations", P5.058, 39th EPS Conference on Plasma Physics, Stockholm. In Europhysics Conference Abstracts Vol. 36F, ISBN 2-914771-79-7, http://ocs.ciemat.es/epsicpp2012pap/pdf/P5.058.pdf

[50] Sauter O, et al. 2002, Plasma Phys. Controlled Fusion 44, 1999.

[51] Turri G, et al., 2008, in Fusion Energy 2008 (Proc. 22nd Int. Conf. Geneva, 2008) (Vienna: IAEA) CD-ROM file EX/P3-06 and http://wwwnaweb.iaea.org/napc/physics/FEC/FEC2008/html/index.htm

[52] Sauter O. et al 1997, Phys. Plasmas 4, 1654.

[53] Houlberg W A, et al, 1997, Phys. Plasmas 4 (9) 3230.

[54] Gribov Y, et al., Progress in the ITER Physics Basis Chapter 8: Plasma operation and control, 2007, Nuclear Fusion 47, S385.

[55] Hertout P, et al, 2011, Proceedings of Symposium of Fusion Technology 2010, Fusion Engineering and Design, vol. 86, pp. 1045-1048.

[56] Urban J, et al., 2012, "Free-boundary equilibrium transport simulations of ITER scenarios under control", P1.019, 39th EPS Conference on Plasma Physics,2012, Stockholm, In Europhysics Conference Abstracts Vol. 36F, ISBN 2-914771-79-7, http://ocs.ciemat.es/epsicpp2012pap/pdf/P1.019.pdf

[57] Artaud J.F. and Kim S.H., 2012, "A new free-boundary equilibrium evolution code, FREEBIE", P4.023, 39th EPS Conference on Plasma Physics, Stockholm, In Europhysics Conference Abstracts Vol. 36F, ISBN 2-914771-79-7; http://ocs.ciemat.es/epsicpp2012pap/pdf/P4.023.pdf

[58] Blum J and Le Foll J, Computer Physics Reports 1 (1984) 465-494.

[59] Fable E, et al., Nucl. Fusion 53 (2013) 033002.

[60] Heikkinen J A, Sipilä S K, et al., 1993, Comp. Phys. Comm. 76 (2), 215.

[61] Dinklage A, Reimer R, et al., 2011, Fusion Sci. Technol. 59, 406. 